\title{
STABILITY AND ERROR ESTIMATES OF LOCAL DISCONTINUOUS GALERKIN METHOD WITH IMPLICIT-EXPLICIT TIME MARCHING FOR SIMULATING WORMHOLE PROPAGATION
}

\author{
Hui GuO ${ }^{1}$, Rui JiA ${ }^{1}$, Lulu Tian ${ }^{1}$ AND YANG YANG ${ }^{2, *}$
}

\begin{abstract}
In this paper, we apply two fully-discrete local discontinuous Galerkin (LDG) methods to the compressible wormhole propagation. We will prove the stability and error estimates of the schemes. Traditional LDG methods use the diffusion term to control of convection term to obtain the stability for some linear equations. However, the variables in wormhole propagation are coupled together and the whole system is highly nonlinear. Therefore, it is extremely difficult to obtain the stability for fully-discrete LDG methods. To fix this gap, we introduce a new auxiliary variable including both the convection and diffusion terms. Moreover, we also construct a special time integration for the porosity, leading to physically relevant numerical approximations and controllable growth rate of the porosity. With a reasonable growth rate, it is possible to handle the time level mismatch in the first-order fully discrete scheme and obtain the stability of the scheme. For the whole system, we will prove that under weak temporal-spatial conditions, the optimal error estimates for the pressure, velocity, porosity and concentration under different norms can be obtained. Numerical experiments are also given to verify the theoretical results.
\end{abstract}

Mathematics Subject Classification. 65M15, 65M60.

Received December 18, 2020. Accepted April 11, 2021.

\section{INTRODUCTION}

The transport and reaction of fluids lead to an evolution of highly conductive flow channels by dissolving the media. These channels are usually named as wormholes. Wormholes help transport the oil and gas in the reservoir to the surface. Therefore, matrix acidization technique plays a significant role in enhancing oil and gas production rates.

The mathematical model of wormhole propagation has been widely studied because of its importance in oil reservoir simulations $[6,7,16,19-21]$. Numerical methods were also investigated intensively. In [38], the chemical-dissolution front instability was numerical established. Later, in [30], the authors analyzed parallel simulation for wormhole propagation. In [1], an alternative numerical-simulation method was given for a modified model. Besides, in [17], the authors presented a 3D simulation of carbonate acidization. A two-phase two-scale

Keywords and phrases. Local discontinuous Galerkin method, implicit-explicit time-marching scheme, stability; error estimate, compressible wormhole propagation.

1 College of Science, China University of Petroleum, Qingdao 266580, P.R. China.

2 Department of Mathematical Sciences, Michigan Technological University, Houghton, MI 49931, USA.

* Corresponding author: yyang7@mtu.edu 
continuum model was developed in [29]. Subsequently, the block-centered finite difference method was considered for compressible and incompressible problems in [12,13], respectively. Moreover, the characteristic splitting mixed finite element method was constructed in [37]. In addition, in [33], the authors investigated the high-order bound-preserving technique. In [11], the mixed finite element method was applied to the wormhole propagation and the stability was well studied. However, this result is only limited to semi-discrete framework. Besides, Li and Rui [14] considered a fully conservative block-centered finite difference method to the model. Since the change of velocity is not small which is a distinct property of wormhole problems, we need to consider methods with high resolution. Therefore, we employ the local discontinuous Galerkin (LDG) method in this paper.

The LDG method was first proposed to solve the convection-diffusion equations in [5], motivated by Bassi and Rebay [2] for studying compressible Navier-Stokes equations. The basic idea is to introduce some axillary variables as the derivatives of the unknown variable then rewrite the original equation into a first order system. Then we can apply the discontinuous Galerkin (DG) method. Therefore, the LDG method enjoys all the advantages of the DG method such as high order accuracy, local solvability and flexibility on hp-adaptivity, etc. It is proved that the stability and optimal error estimates for semi-discrete scheme can be obtained if suitable numerical fluxes can be chosen $[31,32,34,35]$.

Recently, the LDG method for wormhole propagation was presented and analyzed in the semi-discrete framework in $[10,22]$. In fact, an essential feature of wormhole dynamics is that, by the action of acids, the porosity changes significantly and irregularly with time. Therefore, the time discretization of porosity has a significant effect on stability and accuracy of the fully-discrete scheme. Unfortunately, explicit time integrations usually suffer from severe time-step restrictions. Hence in this paper, we will consider the IMEX time integration so that we can take lager time steps, which is one of the main advantages of the IMEX method. Besides, the IMEX time integration has good performance in obtaining elliptic-type algebraic system so that the full scheme can be solved by a variety of standard iterative methods efficiently. In [23-25], the authors incorporated the IMEX time integrations with LDG methods for convection-diffusion problems with linear diffusion terms, yielding outstanding good stability and accuracy. The basic idea was to establish the relationship between the auxiliary variable and the derivative of the primitive variable, then to use the diffusion term to control the convection term. For problems with nonlinear diffusion terms, the stability was demonstrated by numerical experiments only, and the theoretical analysis was totally missing [28]. Later, the IMEX scheme was further applied to incompressible miscible displacements in [26]. However, only error estimates were discussed and the stability was totally missing. The time-dependent incompressible fluid flow problem was also discussed in [27].

One crucial contribution of this paper is that we obtain the stability with mild time step restrictions for the LDG method for wormhole propagation. The analysis is highly nontrivial since the system is highly nonlinear and all the variables are coupled together. In [11], the authors constructed the mixed methods and studied the stability of the semi-discrete scheme. Later, in [13], the authors developed the blocked finite difference methods and investigated the stability. To the best knowledge of the authors, no previous works discussed the stability of LDG methods for wormhole propagation. The stability of the LDG method is more difficult since we need to deal with the inter-element discontinuity and handle the time level mismatch in a fully-discrete scheme. Since the wormhole propagation model is nonlinear, it is impossible to follow the previous works given in [23-25] to obtain the stability.

In this paper, we will handle three main issues in theoretical analysis. Firstly, we control the change of porosity with time. To do so, we define a cut-off operator of the concentration of the solution, keeping the accuracy in the mean time. To construct second-order time integrations and apply the cut-off operator, we extrapolate the concentration by using the values at previous two time steps. In contrast to other second-order time integrations, such as Crank-Nicolson scheme, the technique can avoid solving a fully nonlinear system and hence reduce the computational complexity significantly. Secondly, we introduce a new variable coupling the convection and diffusion terms. This strategy plays a vital role in obtaining stability. In many previous stability analysis for LDG methods, we use the auxiliary variable in the diffusion term to control the jump and gradient of the target unknown variable, see e.g. [23-26]. However, the variables in wormhole propagation are coupled together and the whole system is highly nonlinear. It is not easy to follow the steps in the above works. In this 
paper, we would follow [11] and define an auxiliary function of velocity and establish its properties. Finally, we do not discretize the porosity in space, but solve an ordinary differential equation (ODE) at each point in the computational domain, leading to physically relevant numerical approximations and controllable growth rate of the porosity. Thanks to the special design of the auxiliary variable and the discretization of the porosity, it is possible to develop the stability of the fully-discrete scheme with backward Euler time integration. However, the above idea is not straight-forward extendable to second-order time discretizations due to the time level mismatch in the stability analysis. Without stability, we can only obtain the error estimates for the secondorder time integration by using a different technique (a priori error estimate), see e.g. $[9,10,26,36]$. In this paper, the stability with mild time step restrictions for first-order scheme will be constructed. The optimal error estimate under $L^{\infty}\left(L^{2}\right)$ norm for both schemes are obtained for concentration, velocity, pressure and porosity under weak temporal-spatial conditions, which is the biggest highlight in our work.

The rest of the paper is organized as follows. In Section 2, we demonstrate the governing equations of the compressible wormhole propagation. In Section 3, we present some preliminaries, including the basic notations, norms and projections to be used throughout the paper. In Section 4, we present two IMEX time integrations. The stability will be proved for the first scheme. The error estimates will be given in Section 5 . Numerical results will be provided to demonstrate the accuracy and capability of the method in Section 6 . We will end with some concluding remarks in Section 7.

\section{Compressible WORMHOLE Propagation}

The model of the wormhole propagation over the computational domain $\Omega=[0,1] \times[0,1]$ reads $[11,14]$ :

$$
\begin{aligned}
\gamma \frac{\partial p}{\partial t}+\frac{\partial \phi}{\partial t}+\nabla \cdot \mathbf{u} & =f \\
\mathbf{u} & =\frac{-\kappa(\phi)}{\mu} \nabla p, \\
\frac{\partial \phi c_{f}}{\partial t}+\nabla \cdot\left(\mathbf{u} c_{f}\right)-\nabla \cdot\left(\phi \mathbf{D}(\mathbf{u}) \nabla c_{f}\right) & =f_{p} c_{f}+f_{I} c_{I}+k_{c} a_{v}\left(c_{s}-c_{f}\right), \\
\frac{\partial \phi}{\partial t} & =\frac{\alpha k_{c} a_{v}\left(c_{f}-c_{s}\right)}{\rho_{s}},
\end{aligned}
$$

where $\mathbf{u}$ is the Darcy velocity, $p$ is the pressure, $\phi$ is the porosity, $\kappa$ is the permeability and $c_{f}$ is the cup-mixing concentration of the acid in the fluid phase, respectively. Moreover, $\gamma$ is a pseudo-compressibility parameter that contributes to minor change of the density of the fluid phase in the dissolution process. $f$ is the external volumetric flow rate including the production rate $f_{p}$ and injection rate $f_{I} . \mu$ is the viscosity. $c_{I}$ is the injected concentration. $\mathbf{D}$ is the effective dispersion tensor, and it is defined as

$$
\mathbf{D}(\mathbf{u})=d_{m} \mathbf{I}+|\mathbf{u}|\left\{\alpha_{l} \mathbf{E}(\mathbf{u})+\alpha_{t}(\mathbf{I}-\mathbf{E}(\mathbf{u}))\right\}
$$

where

$$
(\mathbf{E}(\mathbf{u}))_{i j}=\frac{u_{i} u_{j}}{|\mathbf{u}|^{2}}, \quad 1 \leq i \leq 2,
$$

and $d_{m}>0$ is the molecular diffusivity. $\alpha_{l}$ and $\alpha_{t}$ are the longitudinal and the transverse dispersivities which are supposed to be positive, respectively. It is easy to see that $\mathbf{D}(\mathbf{u})$ is positive definite, and hence invertible. $k_{c}$ is the local mass-transfer coefficient and $c_{s}$ is the concentration of the acid at the fluid-solid interface. The variable $c_{s}$ and $c_{f}$ have the following relationship

$$
c_{s}=\frac{c_{f}}{1+k_{s} / k_{c}},
$$


where $k_{s}$ is the surface reaction rate. The relationship between the porosity $\phi$ and the permeability $\kappa$ is established by the Carman-Kozeny correlation [18]

$$
\frac{\kappa}{\kappa_{0}}=\frac{\phi}{\phi_{0}}\left(\frac{\phi\left(1-\phi_{0}\right)}{\phi_{0}(1-\phi)}\right)^{2},
$$

where $\phi_{0}$ and $\kappa_{0}$ are the initial porosity and permeability, respectively. Clearly, $\kappa$ is a function of $\phi$ and

$$
\frac{1}{\kappa(\phi)}=\kappa^{-1}(\phi)=\frac{\phi_{0}}{\phi \kappa_{0}}\left(\frac{\phi_{0}(1-\phi)}{\phi\left(1-\phi_{0}\right)}\right)^{2} .
$$

In (2.4), $\alpha$ is the dissolving power of the acid, $\rho_{s}$ is the density of the solid phase, $a_{v}$ is the interfacial area available for reaction per unit volume of the medium and it can be computed from porosity and permeability as

$$
\frac{a_{v}}{a_{0}}=\frac{\phi}{\phi_{0}} \sqrt{\frac{\kappa_{0} \phi}{\kappa \phi_{0}}}=\frac{1-\phi}{1-\phi_{0}},
$$

where $a_{0}$ is the initial interfacial area. Furthermore, the initial values are given as

$$
c_{f}(x, y, 0)=c_{0}(x, y), \quad p(x, y, 0)=p_{0}(x, y), \quad \phi(x, y, 0)=\phi_{0}(x, y) .
$$

For simplicity, we consider periodic boundary conditions in this paper. The problem with homogeneous Neumann boundary can be analyzed in the same way with some minor changes, and we thus omit it.

Finally, we would like to make the following hypotheses $(\mathrm{H})$ for the problem.

(1) $0<\phi_{*} \leq \phi(x, y, t) \leq \phi^{*}<1$.

(2) $\gamma, \alpha, \rho_{s}, \mu, k_{c}$, and $k_{s}$ are all given positive constants, and $0<\phi_{0 *} \leq \phi_{0} \leq \phi_{0}^{*}<1,0<a_{0 *} \leq a_{0} \leq a_{0}^{*}$.

(3) $c_{f}, c_{f}, \mathbf{u}$ and $\mathbf{s}$ are uniformly bounded in $R^{2} \times[0, T]$.

The following lemma follows from direct computation, hence we only demonstrate the result and skip the proof.

Lemma 2.1. $a_{v}(\phi)$ and $\kappa^{-1}(\phi)$ are Lipschitz continuous, i.e. there exists $C$ such that

$$
\left|a_{v}\left(\phi_{1}\right)-a_{v}\left(\phi_{2}\right)\right| \leq C\left|\phi_{1}-\phi_{2}\right|, \quad\left|\kappa^{-1}\left(\phi_{1}\right)-\kappa^{-1}\left(\phi_{2}\right)\right| \leq C\left|\phi_{1}-\phi_{2}\right| .
$$

\section{Preliminaries}

In this section, we will demonstrate some preliminary results that will be used throughout the paper.

\subsection{Basic notations}

We first construct a rectangular partition $\Omega_{h}$ of $\Omega=[0,1] \times[0,1]$. Let $0=x_{\frac{1}{2}}<x_{\frac{3}{2}}<\cdots<x_{N_{x}+\frac{1}{2}}=1$ and $0=y_{\frac{1}{2}}<y_{\frac{3}{2}}<\cdots<y_{N_{y}+\frac{1}{2}}=1$ be the grid points in $x$ and $y$ directions, respectively. Denote the $i, j$-th cell as

$$
K_{i j}=I_{i} \times J_{j}, \quad i=1, \cdots, N_{x}, j=1, \cdots, N_{y},
$$

where $I_{i}=\left[x_{i-\frac{1}{2}}, x_{i+\frac{1}{2}}\right]$ and $J_{j}=\left[y_{j-\frac{1}{2}}, y_{j+\frac{1}{2}}\right]$. Then $\Omega_{h}=\left\{K_{i j}\right\}$. Moreover, we define $h_{i}^{x}=x_{i+\frac{1}{2}}-x_{i-\frac{1}{2}}$, $h_{j}^{y}=y_{j+\frac{1}{2}}-y_{j-\frac{1}{2}}$ and $h=\max _{i, j}\left(h_{i}^{x}, h_{j}^{y}\right)$. In this paper, we assume the partition is quasi-uniform, i.e. there exists a positive constant $\lambda$ such that $\min _{i, j}\left\{h_{i}^{x}, h_{j}^{y}\right\} \leq h \leq \lambda \min _{i, j}\left\{h_{i}^{x}, h_{j}^{y}\right\}$.

The finite element space is chosen as

$$
W_{h}^{k}=\left\{z:\left.z\right|_{K} \in Q^{k}(K), \forall K \in \Omega_{h}\right\},
$$


where $Q^{k}(K)$ denotes the space of tensor product polynomials of degree at most $k$ in $K$. The set of all element interfaces are denoted by $\Gamma_{h}$ and $\Gamma_{0}=\Gamma_{h} \backslash \partial \Omega$. Define $\boldsymbol{\beta}=(1,1)^{T}$ to be a fixed vector that is not parallel to any normals of the element interfaces. Given $\mathcal{E} \in \Gamma_{0}$, it is shared by two elements $K_{\ell}$ and $K_{r}$, where $\boldsymbol{\beta} \cdot \mathbf{n}_{\ell}>0$, and $\boldsymbol{\beta} \cdot \mathbf{n}_{r}<0$, with $\mathbf{n}_{\ell}$ and $\mathbf{n}_{r}$ being the outward normals of $K_{\ell}$ and $K_{r}$. For any $z \in W_{h}^{k}, z^{-}$and $z^{+}$represent the values of $z$ taken from $K_{\ell}$ and $K_{r}$, respectively. Furthermore, we give the jump as $[z]=z^{+}-z^{-}$. Moreover, for $\mathbf{s} \in \mathbf{W}_{h}^{k}=W_{h}^{k} \times W_{h}^{k}$, we define $\mathbf{s}^{+}$and $\mathbf{s}^{-}$and [s] analogously. We refer readers to [10] for more details.

We use $L^{p}(K)$ for the standard $L^{p}$ space over $K$, with $1 \leq p \leq \infty$, and define the corresponding norm as $\|\cdot\|_{p, K}$. For simplicity, if $p=2$ or $K=\Omega$, then the corresponding subscript will be omitted. In addition, we define $\|\cdot\|_{\Gamma_{K}}^{2}=\langle\cdot, \cdot\rangle_{\partial K}$ where $\langle\cdot, \cdot\rangle_{\partial_{K}}$ is an inner product which will be defined in (3.1). Finally, we denote $\|\cdot\|_{\Gamma_{h}}^{2}=\sum_{K}\|\cdot\|_{\Gamma_{K}}^{2}$. Throughout this paper, $C$ is used as a generic constant independent of mesh size and time step which may have different values at different occurrences. Moreover, $\varepsilon$ is a sufficiently small positive constant.

\subsection{Projections}

Several special projections will be used in this paper. Before defining the projection, we give the classical inverse property [4].

Lemma 3.1. Assume $u \in W_{h}^{k}$, then there exists a positive constant $C$ independent of $h$ and $u$ such that

$$
h\|u\|_{\infty, K}+h^{1 / 2}\|u\|_{\Gamma_{K}} \leq C\|u\|_{K} .
$$

Next we give some inner products

$$
(u, v)_{K}=\int_{K} u v \mathrm{~d} x \mathrm{~d} y, \quad(\mathbf{u}, \mathbf{v})_{K}=\int_{K} \mathbf{u} \cdot \mathbf{v} \mathrm{d} x \mathrm{~d} y, \quad\langle u, v\rangle_{\partial K}=\int_{\partial K} u v \mathrm{~d} s .
$$

Then we define $P^{+}$into $W_{h}^{k}$ which is, for each cell $K$

$$
\begin{aligned}
\left(P^{+} u-u, v\right)_{K} & =0, & & \forall v \in Q^{k-1}(K), \\
\int_{J_{j}}\left(P^{+} u-u\right)\left(x_{i-\frac{1}{2}}, y\right) v(y) \mathrm{d} y & =0, & & \forall v \in P^{k-1}\left(J_{j}\right), \\
\int_{I_{i}}\left(P^{+} u-u\right)\left(x, y_{j-\frac{1}{2}}\right) v(x) \mathrm{d} x & =0, & & \forall v \in P^{k-1}\left(I_{i}\right), \\
\left(P^{+} u-u\right)\left(x_{i-\frac{1}{2}}, y_{j-\frac{1}{2}}\right) & =0, & &
\end{aligned}
$$

where $P^{k}(I)$ denotes the polynomials of degree $k$ over the interval $I$. Moreover, we also define $\Pi_{x}^{-}$and $\Pi_{y}^{-}$into $W_{h}^{k}$ which are, for each cell $K$,

$$
\begin{aligned}
& \left(\Pi_{x}^{-} u-u, v_{x}\right)_{K}=0, \quad \forall v \in Q^{k}(K), \quad \int_{J_{j}}\left(\Pi_{x}^{-} u-u\right)\left(x_{i+\frac{1}{2}}, y\right) v(y) \mathrm{d} y=0, \quad \forall v \in P^{k}\left(J_{j}\right), \\
& \left(\Pi_{y}^{-} u-u, v_{y}\right)_{K}=0, \quad \forall v \in Q^{k}(K), \quad \int_{I_{i}}\left(\Pi_{y}^{-} u-u\right)\left(x, y_{j+\frac{1}{2}}\right) v(x) \mathrm{d} x=0, \quad \forall v \in P^{k}\left(I_{i}\right),
\end{aligned}
$$

as well as a vectored-valued projection $\Pi^{-}=\Pi_{x}^{-} \otimes \Pi_{y}^{-}$.

The following lemma gives the error of the projections [4].

Lemma 3.2. Suppose $w \in H^{k+1}(\Omega), k \geq 0$, then for any project $P_{h}$, which is either $P^{+}, \Pi_{x}^{-}$or $\Pi_{y}^{-}$, we have

$$
\left\|w-P_{h} w\right\|+h^{1 / 2}\left\|w-P_{h} w\right\|_{\Gamma_{h}} \leq C h^{k+1} .
$$


Then the projection $P^{+}$on the Cartesian meshes has the following superconvergence property [3].

Lemma 3.3. Suppose $w \in H^{k+2}(\Omega)$, then for any $K$ and $\boldsymbol{\rho} \in \mathbf{W}_{h}$ we have

$$
\left|\left(w-P^{+} w, \nabla \cdot \boldsymbol{\rho}\right)_{K}-\left\langle w-P^{+} w, \boldsymbol{\rho} \cdot \mathbf{n}_{\mathbf{K}}\right\rangle_{\partial K}\right| \leq C h^{k+1}\|w\|_{k+2}\|\boldsymbol{\rho}\|_{K},
$$

where $\mathbf{n}_{\mathbf{K}}$ is the outward normal of $K$, and the bounding constant $C>0$ is independent of $K$ and $h$.

Before we finish this section, we would like to demonstrate the following lemma whose proof was given in $[15]$.

Lemma 3.4. Let $u \in C^{k+1}(\Omega)$ and $\Pi u \in W_{h}^{k}$. Suppose $\|u-\Pi u\| \leq C h^{\kappa}$ for some positive constant $C$ and $\kappa \leq k+1$. Then

$$
h\|u-\Pi u\|_{\infty}+h^{1 / 2}\|u-\Pi u\|_{\Gamma_{h}} \leq C h^{\kappa},
$$

where $C$ is independent of $h$.

We follow [8] to state the discrete Gronwall's inequality.

Lemma 3.5. Assume $\left\{A_{i}\right\}$ and $\left\{a_{i}\right\}$ are non-negative real number sequences, $q \geq 0$. If for each $i \in \mathbb{N}$, we have

$$
a_{i} \leq A_{i}+\sum_{j=1}^{i-1} q a_{j},
$$

then

$$
a_{i} \leq A_{i}+e^{i q} \sum_{j=1}^{i-1} q A_{j} .
$$

\section{IMEX-LDG SCHEMES}

In this section, we proceed to present the LDG scheme. We will start from the semi-discrete scheme and then provide two fully-discrete LDG schemes.

\subsection{Semi-discrete LDG scheme}

Applying (2.6)-(2.9), we can rewrite the nonlinear system (2.1)-(2.4) into

$$
\begin{aligned}
\gamma \frac{\partial p}{\partial t}+\frac{\partial \phi}{\partial t}+\nabla \cdot \mathbf{u} & =f \\
\mathbf{u} & =\frac{-\kappa(\phi)}{\mu} \nabla p, \\
\frac{\partial \phi c_{f}}{\partial t}+\nabla \cdot \mathbf{s}+A a_{v}(\phi) c_{f} & =f_{p} c_{f}+f_{I} c_{I}, \\
\mathbf{s} & =\mathbf{u} c_{f}-\phi \mathbf{D}(\mathbf{u}) \nabla c_{f}, \\
\frac{\partial \phi}{\partial t} & =B a_{v}(\phi) c_{f},
\end{aligned}
$$

where $A=\frac{k_{c} k_{s}}{k_{c}+k_{s}}, B=\frac{\alpha k_{c} k_{s}}{\rho_{s}\left(k_{c}+k_{s}\right)}$ and $a_{v}(\phi)=\frac{a_{0}(1-\phi)}{1-\phi_{0}}$. Here we introduced a new axillary variable $\mathbf{s}$ which is crucial in obtaining the stability of the scheme. This idea was first introduced in [11] to obtain the stability for semi-discrete mixed method for wormhole propagation. In addition, we define an auxiliary function of velocity as

$$
\mathbf{M}_{\mathbf{e}}(\mathbf{u})=(\mathbf{D}(\mathbf{u}))^{-1} \mathbf{u}
$$


and the cut-off operator $\mathcal{M}$ for velocity as

$$
\mathcal{M}(\mathbf{u}):=\left\{\begin{array}{cl}
\mathbf{u}, & \text { if }|\mathbf{u}| \leq S \\
S \mathbf{u} /|\mathbf{u}|, & \text { if }|\mathbf{u}|>S
\end{array}\right.
$$

where $S$ is a given large positive constant. The LDG discretization for the wormhole propagation (4.1)-(4.4) is now as follows: Find $c_{h}, p_{h}, \in W_{h}^{k}$ and $\mathbf{s}_{h}, \mathbf{u}_{h} \in \mathbf{W}_{h}^{k}$ such that for any $\zeta, v, \in W_{h}^{k}, \boldsymbol{\theta}, \mathbf{w} \in \mathbf{W}_{\mathbf{h}}^{\mathbf{k}}$, the following equations are satisfied

$$
\begin{aligned}
\left(\gamma \frac{\partial p_{h}}{\partial t}, \zeta\right)_{K}+\left(\frac{\partial \phi_{h}}{\partial t}, \zeta\right)_{K} & =\mathcal{L}_{K}^{d}\left(\mathbf{u}_{\mathbf{h}}, \zeta\right)+(f, \zeta)_{K}, \\
\left(\frac{\mu}{\kappa\left(\phi_{h}\right)} \mathbf{u}_{\mathbf{h}}, \boldsymbol{\theta}\right)_{K} & =D_{K}\left(p_{h}, \boldsymbol{\theta}\right), \\
\left(\left(\phi_{h} c_{h}\right)_{t}, v\right)_{K} & =\mathcal{L}_{K}^{d}\left(\mathbf{s}_{\mathbf{h}}, v\right)+\left(f_{p} c_{h}+f_{I} c_{I}, v\right)_{K}-\left(A a_{v}\left(\phi_{h}\right) c_{h}, v\right)_{K}, \\
\left(\left(\phi_{h} \mathbf{D}\left(\mathbf{u}_{\mathbf{h}}^{\mathbf{M}}\right)\right)^{-1} \mathbf{s}_{\mathbf{h}}, \mathbf{w}\right)_{K} & =\left(\left(\phi_{h}\right)^{-1} \mathbf{M}_{\mathbf{e}}\left(\mathbf{u}_{\mathbf{h}}\right) c_{h}, \mathbf{w}\right)_{K}+D_{K}\left(c_{h}, \mathbf{w}\right),
\end{aligned}
$$

where

$$
\mathcal{L}_{K}^{d}(\mathbf{s}, v)=(\mathbf{s}, \nabla v)_{K}-\left\langle\widehat{\mathbf{s}} \cdot \boldsymbol{\nu}_{K}, v\right\rangle_{\partial K}, \quad \text { and } \quad \mathcal{D}_{K}(c, \mathbf{w})=(c, \nabla \cdot \mathbf{w})_{K}-\left\langle\widehat{c}, \mathbf{w} \cdot \boldsymbol{\nu}_{K}\right\rangle_{\partial K},
$$

with $\boldsymbol{\nu}_{K}$ being the unit outer normal of $K$ and $\mathbf{u}_{\mathbf{h}}^{\mathrm{M}}=\mathcal{M}\left(\mathbf{u}_{\mathbf{h}}\right)$. The time evolution of the porosity (4.5) is given as

$$
\frac{\partial \phi_{h}}{\partial t}=B a_{v}\left(\phi_{h}\right) \bar{c}_{h}
$$

where $\bar{c}_{h}=\max \left(0, \min \left(c_{h}, 1\right)\right)$.

Remark 4.1. Notice that $\phi_{h}$ is not in the finite element spaces and the time integration is computed at each point. For given $(x, y), \phi_{h}(x, y)$ is obtained by solving an ODE numerically. However, in actual simulations, we only need to calculate the value of the Gaussian quadrature points.

The hat terms are the numerical fluxes. In this paper, we take

$$
\widehat{\mathbf{s}_{h}}=\mathbf{s}_{h}^{-}, \quad \widehat{c_{h}}=c_{h}^{+}, \quad \widehat{\mathbf{u}_{h}}=\mathbf{u}_{h}^{-}, \quad \widehat{p_{h}}=p_{h}^{+}
$$

Moreover, we define

$$
(u, v)=\sum_{K \in \Omega_{h}}(u, v)_{K}, \quad(\mathbf{u}, \mathbf{v})=\sum_{K \in \Omega_{h}}(\mathbf{u}, \mathbf{v})_{K}, \quad \mathcal{L}^{d}(\mathbf{s}, v)=\sum_{K \in \Omega_{h}} \mathcal{L}_{K}^{d}(\mathbf{s}, v), \quad \mathcal{D}(c, \mathbf{w})=\sum_{K \in \Omega_{h}} \mathcal{D}_{K}(c, \mathbf{w})
$$

With integration by parts, it is easy to check that for any $v$ and $\mathbf{w}$, we have

$$
\mathcal{L}^{d}(\mathbf{w}, v)+\mathcal{D}(v, \mathbf{w})=0
$$

\subsection{Fully-discrete LDG schemes}

Let $\left\{t^{n}=n \tau\right\}_{n=0}^{M}$ be a uniform partition of the time interval $[0, T]$, with time step size $\tau=T / M$. However, this assumption of uniform partition is not essential. In this paper, we consider two IMEX time integrations coupled with LDG spatial discretization. For convenience, we use the same notations. 


\subsubsection{First-order time integration}

The first-order time integration, denoted as IMEX-LDG $(k, 1)$, will be constructed. Given the numerical solutions $c_{h}^{n}, p_{h}^{n}, \phi_{h}^{n}, \mathbf{u}_{\mathbf{h}}^{\mathbf{n}}, \mathbf{s}_{\mathbf{h}}^{\mathbf{n}}$ at time level $n$, we would compute $\phi_{h}^{n+1}$ by

$$
\frac{\phi_{h}^{n+1}-\phi_{h}^{n}}{\tau}=B a_{v}\left(\phi_{h}^{n+1}\right) \bar{c}_{h}^{n} .
$$

Then we can obtain $p_{h}^{n+1}, \mathbf{u}_{\mathbf{h}}^{\mathbf{n}+\mathbf{1}}$ by using

$$
\begin{aligned}
\left(\gamma \frac{p_{h}^{n+1}-p_{h}^{n}}{\tau}, \zeta\right)+\left(\frac{\phi_{h}^{n+1}-\phi_{h}^{n}}{\tau}, \zeta\right) & =\mathcal{L}^{d}\left(\mathbf{u}_{\mathbf{h}}^{\mathbf{n}+\mathbf{1}}, \zeta\right)+\left(f^{n}, \zeta\right), \\
\left(\frac{\mu}{\kappa\left(\phi_{h}^{n+1}\right)} \mathbf{u}_{\mathbf{h}}^{\mathbf{n}+\mathbf{1}}, \boldsymbol{\theta}\right) & =\mathcal{D}\left(p_{h}^{n+1}, \boldsymbol{\theta}\right),
\end{aligned}
$$

for any $\zeta \in W_{h}^{k}, \boldsymbol{\theta} \in \mathbf{W}_{\mathbf{h}}^{\mathbf{k}}$. Finally, $c_{h}^{n+1}, \mathbf{s}_{\mathbf{h}}^{\mathbf{n}+\mathbf{1}}$ can be find by the variational form

$$
\begin{aligned}
\left(\frac{\phi_{h}^{n+1} c_{h}^{n+1}-\phi_{h}^{n} c_{h}^{n}}{\tau}, v\right) & =\mathcal{L}^{d}\left(\mathbf{s}_{\mathbf{h}}^{\mathbf{n}+\mathbf{1}}, v\right)-\left(A a_{v}\left(\phi_{h}^{n}\right) c_{h}^{n}, v\right)+\left(f_{p}^{n} c_{h}^{n}+f_{I}^{n} c_{I}^{n}, v\right), \\
\left(\left(\phi_{h}^{n+1} \mathbf{D}\left(\mathbf{u}_{\mathbf{h}}^{\mathbf{n}+\mathbf{1}, \mathbf{M}}\right)\right)^{-1} \mathbf{s}_{\mathbf{h}}^{\mathbf{n}+\mathbf{1}}, \mathbf{w}\right) & =\left(\left(\phi_{h}^{n+1}\right)^{-1} \mathbf{M}_{\mathbf{e}}\left(\mathbf{u}_{\mathbf{h}}^{\mathbf{n}+\mathbf{1}}\right) c_{h}^{n+1}, \mathbf{w}\right)+\mathcal{D}\left(c_{h}^{n+1}, \mathbf{w}\right),
\end{aligned}
$$

for any $v \in W_{h}^{k}, \mathbf{w} \in \mathbf{W}_{\mathbf{h}}^{\mathbf{k}}$. The initial solutions are

$$
\phi_{h}(x, y, 0)=\phi(x, y, 0), \quad c_{h}(x, y, 0)=P^{+} c_{0}, \quad p_{h}(x, y, 0)=P^{+} p_{0},
$$

\subsubsection{Second-order time integration}

We proceed to construct the second-order time integration, namely IMEX-LDG( $(k, 2)$. For any $n \geq 1$, suppose the numerical solutions at $t^{n}, t^{n-1}$ are available, we first get $\phi_{h}^{n+1}$ such that

$$
\frac{\phi_{h}^{n+1}-\phi_{h}^{n}}{\tau}=\frac{1}{2} B a_{v}\left(\phi_{h}^{n+1}\right) \bar{c}_{h}^{n+1, *}+\frac{1}{2} B a_{v}\left(\phi_{h}^{n}\right) \bar{c}_{h}^{n},
$$

where

$$
c_{h}^{n+1, *}=\left\{\begin{array}{cc}
2 c_{h}^{n}-c_{h}^{n-1}, & n \geq 1, \\
c_{h}^{0}, & n=0 .
\end{array}\right.
$$

Then, we calculate $p_{h}^{n+1}, \mathbf{u}_{\mathbf{h}}^{\mathbf{n}+\mathbf{1}}$ by

$$
\begin{aligned}
\left(\gamma \frac{p_{h}^{n+1}-p_{h}^{n}}{\tau}, \zeta\right)+\left(\frac{\phi_{h}^{n+1}-\phi_{h}^{n}}{\tau}, \zeta\right) & =\frac{3}{4} \mathcal{L}^{d}\left(\mathbf{u}_{\mathbf{h}}^{\mathbf{n}+\mathbf{1}}, \zeta\right)+\frac{1}{4} \mathcal{L}^{d}\left(\mathbf{u}_{\mathbf{h}}^{\mathbf{n}-\mathbf{1}}, \zeta\right)+\frac{3}{2}\left(f^{n}, \zeta\right)-\frac{1}{2}\left(f^{n-1}, \zeta\right), \\
\left(\frac{\mu}{\kappa\left(\phi_{h}^{n+1}\right)} \mathbf{u}_{\mathbf{h}}^{\mathbf{n}+\mathbf{1}}, \boldsymbol{\theta}\right) & =\mathcal{D}\left(p_{h}^{n+1}, \boldsymbol{\theta}\right),
\end{aligned}
$$

for any $\zeta \in W_{h}^{k}, \boldsymbol{\theta} \in \mathbf{W}_{\mathbf{h}}^{\mathbf{k}}$. Finally, we can find $c_{h}^{n+1}, \mathbf{s}_{\mathbf{h}}^{\mathbf{n}+\mathbf{1}}$ by

$$
\begin{aligned}
\left(\frac{\phi_{h}^{n+1} c_{h}^{n+1}-\phi_{h}^{n} c_{h}^{n}}{\tau}, v\right)= & \frac{3}{4} \mathcal{L}^{d}\left(\mathbf{s}_{\mathbf{h}}^{\mathbf{n}+\mathbf{1}}, v\right)+\frac{1}{4} \mathcal{L}^{d}\left(\mathbf{s}_{\mathbf{h}}^{\mathbf{n}-\mathbf{1}}, v\right)-\frac{3}{2}\left(A a_{v}\left(\phi_{h}^{n}\right) c_{h}^{n}, v\right) \\
& +\frac{1}{2}\left(A a_{v}\left(\phi_{h}^{n-1}\right) c_{h}^{n-1}, v\right)
\end{aligned}
$$




$$
\begin{aligned}
& +\frac{3}{2}\left(f_{p}^{n} c_{h}^{n}+f_{I}^{n} c_{I}^{n}, v\right)-\frac{1}{2}\left(f_{p}^{n-1} c_{h}^{n-1}+f_{I}^{n-1} c_{I}^{n-1}, v\right), \\
\left(\left(\phi_{h}^{n+1} \mathbf{D}\left(\mathbf{u}_{\mathbf{h}}^{\mathbf{n}+\mathbf{1}, \mathbf{M}}\right)\right)^{-1} \mathbf{s}_{\mathbf{h}}^{\mathbf{n}+\mathbf{1}}, \mathbf{w}\right)= & \left(\left(\phi_{h}^{n+1}\right)^{-1} \mathbf{M}_{\mathbf{e}}\left(\mathbf{u}_{\mathbf{h}}^{\mathbf{n}+\mathbf{1}}\right) c_{h}^{n+1}, \mathbf{w}\right)+\mathcal{D}\left(c_{h}^{n+1}, \mathbf{w}\right),
\end{aligned}
$$

for all $v \in W_{h}^{k}, \mathbf{w} \in \mathbf{W}_{\mathbf{h}}^{\mathbf{k}}$.

To complete the scheme, we need initial solutions at two time levels. We take the solutions at $t^{0}$ from (4.19) and the solution at $t^{1}$ is from the first-order time integration introduced in the previous subsection.

\subsection{Stability analysis}

In this subsection, we will prove the stability of the IMEX-LDG scheme. We first present some useful properties of $\mathbf{D}(\mathbf{u})$ and $\mathbf{M}_{\mathbf{e}}(\mathbf{u})$ given in [11].

Lemma 4.2. Let $\mathbf{D}(\mathbf{u})$ be defined in (2.5) and $d_{m}>0$. Suppose that $\alpha_{l}$ and $\alpha_{t}$ are non-negative functions of $x$ and are uniformly bounded, i.e. $\alpha_{l}(x) \leq \alpha_{l}^{*}$ and $\alpha_{t}(x) \leq \alpha_{t}^{*}$. Then for any $\mathbf{u}, \mathbf{v} \in R^{d}$,

$$
\begin{aligned}
\left|\mathbf{D}(\mathbf{u})^{-\mathbf{1}} \mathbf{v}\right| & \leq\left(d_{m}+\min \left(\alpha_{l}, \alpha_{t}\right)|\mathbf{u}|\right)^{-1}|\mathbf{v}| \leq d_{m}^{-1}|\mathbf{v}| \\
\left|\mathbf{D}(\mathbf{u})^{-1} \mathbf{v}\right| & \geq\left(d_{m}+\max \left(\alpha_{l}^{*}, \alpha_{t}^{*}\right)|\mathbf{u}|\right)^{-1}|\mathbf{v}| \\
\left|\mathbf{D}(\mathbf{u})^{-\mathbf{1}}-\mathbf{D}(\mathbf{v})^{-\mathbf{1}}\right| & \leq d_{m}^{-2}\left(7 \alpha_{t}^{*}+6 \alpha_{l}^{*}\right) d^{3 / 2}|\mathbf{u}-\mathbf{v}|
\end{aligned}
$$

where $d$ is the dimension of domain $\Omega$.

Lemma 4.3. Let $\mathbf{M}_{\mathbf{e}}(\mathbf{u})$ be defined in (4.6) and $d_{m}>0$. Suppose that $\alpha_{l}$ and $\alpha_{t}$ are positive functions of $x$ and $0<\alpha_{l^{*}} \leq \alpha_{l}(x) \leq \alpha_{l}^{*}$ and $0<\alpha_{t^{*}} \leq \alpha_{t}(x) \leq \alpha_{t}^{*}$. Then for $\mathbf{u}, \mathbf{v} \in R^{d}$,

$$
\begin{aligned}
\left|\mathbf{M}_{\mathbf{e}}(\mathbf{u})\right| & \leq\left(\min \left(\alpha_{l^{*}}, \alpha_{t^{*}}\right)\right)^{-1} \\
\left|\mathbf{M}_{\mathbf{e}}(\mathbf{u})-\mathbf{M}_{\mathbf{e}}(\mathbf{v})\right| & \leq L_{M}|\mathbf{u}-\mathbf{v}|
\end{aligned}
$$

where $L_{M}=d_{m}^{-1}+\left(d_{m} \min \left(\alpha_{l^{*}}, \alpha_{t^{*}}\right)\right)^{-1}\left(7 \alpha_{t}^{*}+6 \alpha_{l}^{*}\right) d^{3 / 2}$ and $d$ is the dimension of domain $\Omega$.

We now prove the stability results for the discrete solutions of the IMEX-LDG schemes.

Theorem 4.4. The discrete porosity $\phi_{h}$ from the $\operatorname{IMEX-LDG(k,1)}$ scheme is bounded, i.e.,

$$
\phi_{0} \leq \phi_{h}^{n} \leq 1-\left(1-\phi_{0}\right) e^{-\psi T}<1,
$$

where $\phi_{0}>0$ is the initial porosity and $\psi=\frac{R}{1-\phi_{0}}$. It also holds that

$$
0 \leq \frac{\phi_{h}^{n+1}-\phi_{h}^{n}}{\tau}<R
$$

where $R=B a_{0}=\frac{\alpha k_{c} k_{s} a_{0}}{\rho_{s}\left(k_{c}+k_{s}\right)}, n \geq 0$. Moreover, the estimates $\phi_{0} \leq \phi_{h}^{n}<1$ and (4.32) are also valid for IMEX-LDG(k,2) schemes provided

$$
\tau \leq \frac{2\left(1-\phi_{0}\right)}{R}
$$

Proof. We first prove for the IMEX-LDG $(k, 1)$ scheme. We use mathematical induction. With the initial solutions taking in (4.19), we have $\phi_{h}^{0}=\phi_{0}$. Then it is easy to see that $\phi_{0} \leq \phi_{h}^{0}<1$ is satisfied. Now assume $\phi_{0} \leq \phi_{h}^{n}<1$, we want to prove $\phi_{0} \leq \phi_{h}^{n+1}<1$. We rewrite (4.14) as

$$
\frac{\phi_{h}^{n+1}-\phi_{h}^{n}}{\tau}=R \frac{1-\phi_{h}^{n+1}}{1-\phi_{0}} \bar{c}_{h}^{n} .
$$


Define $\beta^{n}=\frac{R}{1-\phi_{0}} \bar{c}_{h}^{n} \tau \geq 0$, then with direct calculation, we have

$$
\phi_{h}^{n+1}=\frac{\beta^{n}+\phi_{h}^{n}}{1+\beta^{n}}<1 .
$$

We can also deduce that $\phi_{h}^{n+1}-\phi_{h}^{n}=\beta^{n}\left(1-\phi_{h}^{n+1}\right) \geq 0$, namely

$$
\phi_{0} \leq \phi_{h}^{n} \leq \phi_{h}^{n+1}<1 .
$$

Now we proceed to prove the more accurate upper bound of $\phi_{h}$. Noticing that

$$
\frac{\phi_{h}^{n+1}-\phi_{h}^{n}}{\tau} \leq \psi\left(1-\phi_{h}^{n+1}\right),
$$

with $\psi=\frac{R}{1-\phi_{0}}$, we obtain

$$
\left(1-\phi_{h}^{n+1}\right) \geq \frac{1}{1+\psi \tau}\left(1-\phi_{h}^{n}\right)
$$

which further yields

$$
\left(1-\phi_{h}^{n}\right) \geq\left(\frac{1}{1+\psi \tau}\right)^{n}\left(1-\phi_{0}\right) \geq\left(e^{-\psi \tau}\right)^{n}\left(1-\phi_{0}\right) \geq e^{-\psi T}\left(1-\phi_{0}\right) .
$$

Therefore,

$$
\phi_{h}^{n} \leq 1-\left(1-\phi_{0}\right) e^{-\psi T}<1 .
$$

Then we can obtain (4.31). With the bounds of $\phi_{h}^{n+1}$ given in (4.34), the proof of (4.32) is straightforward.

Next we prove for the IMEX-LDG $(k, 2)$ scheme. We also assume $\phi_{0} \leq \phi_{h}^{n}<1$ and rewrite (4.20) as

$$
\phi_{h}^{n+1}=\frac{1}{2} R \frac{1-\phi_{h}^{n+1}}{1-\phi_{0}} \bar{c}_{h}^{n+1, *} \tau+\frac{1}{2} R \frac{1-\phi_{h}^{n}}{1-\phi_{0}} \bar{c}_{h}^{n} \tau+\phi_{h}^{n} .
$$

We define $\beta^{n+1, *}=R \frac{\bar{c}_{h}^{n+1, *}}{1-\phi_{0}} \tau \geq 0$ and $\beta^{n}=R \frac{\bar{c}_{h}^{n}}{1-\phi_{0}} \tau \geq 0$. Under (4.33), we have $\beta^{n} \leq 2$. Then with direct calculation, we can obtain

$$
\phi_{h}^{n+1}=\frac{\frac{1}{2} \beta^{n+1, *}+\frac{1}{2} \beta^{n}+\left(1-\frac{1}{2} \beta^{n}\right) \phi_{h}^{n}}{1+\frac{1}{2} \beta^{n+1, *}}<1,
$$

which further yields $1>\phi_{h}^{n} \geq \phi_{0}$. The estimate in (4.32) is straightforward.

Then we can state the stability of the $\operatorname{IMEX-LDG}(k, 1)$ scheme.

Theorem 4.5. The approximate solutions of IMEX-LDG(k,1) scheme satisfy

$$
\begin{gathered}
\left\|p_{h}^{n}\right\|^{2}+\tau \sum_{m=1}^{n}\left\|\mathbf{u}_{\mathbf{h}}^{\mathbf{m}}\right\|^{2} \leq C \tau \sum_{m=1}^{n}\left\|f^{m-1}\right\|^{2}+C R^{2}+C\left\|p_{h}^{0}\right\|^{2}, \\
\left\|c_{h}^{n}\right\|^{2}+\tau \sum_{m=1}^{n}\left\|\mathbf{s}_{\mathbf{h}}^{\mathbf{m}}\right\|^{2} \leq C \tau \sum_{m=1}^{n}\left\|f_{I}^{m-1} c_{I}^{m-1}\right\|^{2}+C\left\|c_{h}^{0}\right\|^{2}
\end{gathered}
$$

where $R=B a_{0}=\frac{\alpha k_{c} k_{s} a_{0}}{\rho_{s}\left(k_{c}+k_{s}\right)}$ and $C \tau \leq \min \left(\frac{\gamma}{2}, \frac{\phi_{0}}{2}\right)$. 
Proof. Taking $\zeta=p_{h}^{n+1}$ in (4.15) and $\boldsymbol{\theta}=\mathbf{u}_{\mathbf{h}}^{\mathbf{n}+\mathbf{1}}$ in (4.16), we obtain

$$
\begin{aligned}
\left(\gamma \frac{p_{h}^{n+1}-p_{h}^{n}}{\tau}, p_{h}^{n+1}\right)+\left(\frac{\phi_{h}^{n+1}-\phi_{h}^{n}}{\tau}, p_{h}^{n+1}\right) & =\mathcal{L}^{d}\left(\mathbf{u}_{\mathbf{h}}^{\mathbf{n}+\mathbf{1}}, p_{h}^{n+1}\right)+\left(f^{n}, p_{h}^{n+1}\right) \\
\left(\frac{\mu}{\kappa\left(\phi_{h}^{n+1}\right)} \mathbf{u}_{\mathbf{h}}^{\mathbf{n}+\mathbf{1}}, \mathbf{u}_{\mathbf{h}}^{\mathbf{n}+\mathbf{1}}\right) & =\mathcal{D}\left(p_{h}^{n+1}, \mathbf{u}_{\mathbf{h}}^{\mathbf{n}+\mathbf{1}}\right)
\end{aligned}
$$

Summing (4.37) and (4.38) and using (4.13), we have

$$
\begin{aligned}
& \frac{\gamma}{2}\left(\left\|p_{h}^{n+1}\right\|^{2}+\left\|p_{h}^{n+1}-p_{h}^{n}\right\|^{2}-\left\|p_{h}^{n}\right\|^{2}\right)+\tau\left\|\left(\frac{\mu}{\kappa\left(\phi_{h}^{n+1}\right)}\right)^{1 / 2} \mathbf{u}_{\mathbf{h}}^{\mathbf{n}+\mathbf{1}}\right\|^{2} \\
& \quad \leq-\tau\left(\frac{\phi_{h}^{n+1}-\phi_{h}^{n}}{\tau}, p_{h}^{n+1}\right)+\tau\left(f^{n}, p_{h}^{n+1}\right) \leq \tau\left(\left\|\frac{\phi_{h}^{n+1}-\phi_{h}^{n}}{\tau}\right\|+\left\|f^{n}\right\|\right)\left\|p_{h}^{n+1}\right\| \\
& \quad \leq \tau\left(\left\|\frac{\phi_{h}^{n+1}-\phi_{h}^{n}}{\tau}\right\|+\left\|f^{n}\right\|\right)\left(\left\|p_{h}^{n}\right\|+\left\|p_{h}^{n+1}-p_{h}^{n}\right\|\right) \leq \tau\left(R^{2}+\left\|p_{h}^{n}\right\|^{2}+\left\|f^{n}\right\|^{2}+\left\|p_{h}^{n+1}-p_{h}^{n}\right\|^{2}\right),
\end{aligned}
$$

where the third step requires triangle inequality and the last step requires Theorem 4.4 and Young's inequality. Then we get

$$
\frac{\gamma}{2}\left\|p_{h}^{n+1}\right\|^{2}-\frac{\gamma}{2}\left\|p_{h}^{n}\right\|^{2}+\tau\left\|\left(\frac{\mu}{\kappa\left(\phi_{h}^{n+1}\right)}\right)^{1 / 2} \mathbf{u}_{\mathbf{h}}^{\mathbf{n}+\mathbf{1}}\right\|^{2} \leq \tau\left(R^{2}+\left\|p_{h}^{n}\right\|^{2}+\left\|f^{n}\right\|^{2}\right)
$$

with $\tau \leq \frac{\gamma}{2}$. We can deduce the lower bound of $\kappa^{-1}\left(\phi_{h}^{n+1}\right)$ through (2.8) with the upper bound of $\phi_{h}^{n+1}$ given in (4.31). Using Lemma 3.5 and summing (4.40) over $n$, we can obtain (4.35) in Theorem 4.5.

We now turn to prove (4.36). Taking $v=c_{h}^{n+1}$ in (4.17) and $\mathbf{w}=\mathbf{s}_{\mathbf{h}}^{\mathbf{n}+\mathbf{1}}$ in (4.18), we have

$$
\begin{aligned}
\left(\frac{\phi_{h}^{n+1} c_{h}^{n+1}-\phi_{h}^{n} c_{h}^{n}}{\tau}, c_{h}^{n+1}\right) & =\mathcal{L}^{d}\left(\mathbf{s}_{\mathbf{h}}^{\mathbf{n}+\mathbf{1}}, c_{h}^{n+1}\right)-\left(A a_{v}\left(\phi_{h}^{n}\right) c_{h}^{n}, c_{h}^{n+1}\right)+\left(f_{p}^{n} c_{h}^{n}+f_{I}^{n} c_{I}^{n}, c_{h}^{n+1}\right), \\
\left(\left(\phi_{h}^{n+1} \mathbf{D}\left(\mathbf{u}_{\mathbf{h}}^{\mathbf{n}+\mathbf{1}, \mathbf{M}}\right)\right)^{-1} \mathbf{s}_{\mathbf{h}}^{\mathbf{n}+\mathbf{1}}, \mathbf{s}_{\mathbf{h}}^{\mathbf{n}+\mathbf{1}}\right) & =\left(\left(\phi_{h}^{n+1}\right)^{-1} \mathbf{M}_{\mathbf{e}}\left(\mathbf{u}_{\mathbf{h}}^{\mathbf{n}+\mathbf{1}}\right) c_{h}^{n+1}, \mathbf{s}_{\mathbf{h}}^{\mathbf{n}+\mathbf{1}}\right)+\mathcal{D}\left(c_{h}^{n+1}, \mathbf{s}_{\mathbf{h}}^{\mathbf{n}+\mathbf{1}}\right) .
\end{aligned}
$$

It is easy to verify that

$$
\begin{aligned}
\left(\phi_{h}^{n+1}\right. & \left.\left(c_{h}^{n+1}-c_{h}^{n}\right), c_{h}^{n+1}\right) \\
& =\frac{1}{2}\left(\phi_{h}^{n+1} c_{h}^{n+1}, c_{h}^{n+1}\right)+\frac{1}{2}\left(\phi_{h}^{n+1}\left(c_{h}^{n+1}-c_{h}^{n}\right),\left(c_{h}^{n+1}-c_{h}^{n}\right)\right)-\frac{1}{2}\left(\phi_{h}^{n+1} c_{h}^{n}, c_{h}^{n}\right) \\
& =\frac{1}{2}\left(\phi_{h}^{n+1} c_{h}^{n+1}, c_{h}^{n+1}\right)+\frac{1}{2}\left(\phi_{h}^{n+1}\left(c_{h}^{n+1}-c_{h}^{n}\right),\left(c_{h}^{n+1}-c_{h}^{n}\right)\right)-\frac{1}{2}\left(\left(\phi_{h}^{n}+\tau B a_{v}\left(\phi_{h}^{n+1}\right) \bar{c}_{h}^{n}\right) c_{h}^{n}, c_{h}^{n}\right) \\
& \geq \frac{1}{2}\left(\phi_{h}^{n+1} c_{h}^{n+1}, c_{h}^{n+1}\right)+\frac{1}{2}\left(\phi_{h}^{n+1}\left(c_{h}^{n+1}-c_{h}^{n}\right),\left(c_{h}^{n+1}-c_{h}^{n}\right)\right)-\frac{1}{2}\left(\phi_{h}^{n} c_{h}^{n}, c_{h}^{n}\right)-\frac{1}{2} \tau R\left(c_{h}^{n}, c_{h}^{n}\right), \quad
\end{aligned}
$$

where we have used the fact that $\phi_{h}^{n+1}=\phi_{h}^{n}+\tau B a_{v}\left(\phi_{h}^{n+1}\right) \bar{c}_{h}^{n}$ in (4.14). Summing (4.41) and (4.42) and using (4.13) to get

$$
\frac{1}{2}\left(\left\|\sqrt{\phi_{h}^{n+1}} c_{h}^{n+1}\right\|^{2}+\left\|\sqrt{\phi_{h}^{n+1}}\left(c_{h}^{n+1}-c_{h}^{n}\right)\right\|^{2}-\left\|\sqrt{\phi_{h}^{n}} c_{h}^{n}\right\|^{2}\right)+\tau\left\|\left(\phi_{h}^{n+1} \mathbf{D}\left(\mathbf{u}_{\mathbf{h}}^{\mathbf{n}+\mathbf{1}, \mathbf{M}}\right)\right)^{-1 / 2} \mathbf{s}_{\mathbf{h}}^{\mathbf{n}+\mathbf{1}}\right\|^{2}
$$




$$
\begin{aligned}
\leq & -\tau\left(\frac{\left(\phi_{h}^{n+1}-\phi_{h}^{n}\right)}{\tau} c_{h}^{n}, c_{h}^{n+1}\right)-\tau\left(A a_{v}\left(\phi_{h}^{n}\right) c_{h}^{n}, c_{h}^{n+1}\right)+\tau\left(f_{p}^{n} c_{h}^{n}+f_{I}^{n} c_{I}^{n}, c_{h}^{n+1}\right) \\
& +\tau\left(\left(\phi_{h}^{n+1}\right)^{-1} \mathbf{M}_{\mathbf{e}}\left(\mathbf{u}_{\mathbf{h}}^{\mathbf{n}+\mathbf{1}}\right) c_{h}^{n+1}, \mathbf{s}_{\mathbf{h}}^{\mathbf{n}+\mathbf{1}}\right)+\frac{1}{2} \tau R\left(c_{h}^{n}, c_{h}^{n}\right) .
\end{aligned}
$$

We estimate the fourth term on the right side of the above equation

$$
\begin{aligned}
\left(\left(\phi_{h}^{n+1}\right)^{-1} \mathbf{M}_{\mathbf{e}}\left(\mathbf{u}_{\mathbf{h}}^{\mathbf{n}+\mathbf{1}}\right) c_{h}^{n+1}, \mathbf{s}_{\mathbf{h}}^{\mathbf{n}+\mathbf{1}}\right) & \leq\left\|c_{h}^{n+1}\right\|\left\|\left(\phi_{h}^{n+1}\right)^{-1} \mathbf{M}_{\mathbf{e}}\left(\mathbf{u}_{\mathbf{h}}^{\mathbf{n}+\mathbf{1}}\right) \mathbf{s}_{\mathbf{h}}^{\mathbf{n}+\mathbf{1}}\right\| \\
& \leq C\left\|c_{h}^{n+1}\right\|\left\|\left(\phi_{h}^{n+1} \mathbf{D}\left(\mathbf{u}_{\mathbf{h}}^{\mathbf{n}+\mathbf{1}, \mathbf{M}}\right)\right)^{-1 / 2} \mathbf{s}_{\mathbf{h}}^{\mathbf{n}+\mathbf{1}}\right\| \\
& \leq C\left(\left\|c_{h}^{n}\right\|+\left\|c_{h}^{n+1}-c_{h}^{n}\right\|\right)\left\|\left(\phi_{h}^{n+1} \mathbf{D}\left(\mathbf{u}_{\mathbf{h}}^{\mathbf{n}+\mathbf{1}, \mathbf{M}}\right)\right)^{-1 / 2} \mathbf{s}_{\mathbf{h}}^{\mathbf{n}+\mathbf{1}}\right\| \\
& \leq C\left(\left\|c_{h}^{n}\right\|^{2}+\left\|c_{h}^{n+1}-c_{h}^{n}\right\|^{2}\right)+\varepsilon \|\left(\phi_{h}^{n+1} \mathbf{D}\left(\mathbf{u}_{\mathbf{h}}^{\mathbf{n}+\mathbf{1}, \mathbf{M}}\right)\right)^{-1 / 2} \mathbf{s}_{\mathbf{h}}^{\mathbf{n}+\mathbf{1} \|^{2}},
\end{aligned}
$$

where in the first step we use Schwarz inequality and in the second step we use the boudedness of $\mathbf{M}_{\mathbf{e}}\left(\mathbf{u}_{\mathbf{h}}^{\mathbf{n}+\mathbf{1}}\right)$ given in (4.29) and the lower bound of $\left(\mathbf{D}\left(\mathbf{u}_{\mathbf{h}}^{\mathbf{n}+\mathbf{1}, \mathbf{M}}\right)\right)^{-\mathbf{1}}$ given in (4.27) while the third step follows from triangle inequality. Thanks to Theorem 4.4 and Young's inequality, and under the condition $C \tau \leq \frac{\phi_{0}}{2}$, we have

$$
\frac{1}{2}\left\|\sqrt{\phi_{h}^{n+1}} c_{h}^{n+1}\right\|^{2}-\frac{1}{2}\left\|\sqrt{\phi_{h}^{n}} c_{h}^{n}\right\|^{2}+\frac{7 \tau}{8}\left\|\left(\phi_{h}^{n+1} \mathbf{D}\left(\mathbf{u}_{\mathbf{h}}^{\mathbf{n}+\mathbf{1}, \mathbf{M}}\right)\right)^{-1 / 2} \mathbf{s}_{\mathbf{h}}^{\mathbf{n}+\mathbf{1}}\right\|^{2} \leq C \tau\left(\left\|c_{h}^{n}\right\|^{2}+\left\|f_{I}^{n} c_{I}^{n}\right\|^{2}\right),
$$

with taking $\varepsilon=\frac{1}{8}$. Summing (4.46) over $n$ and using Lemma 3.5, we finish the proof.

\section{ERROR ESTIMATE}

\subsection{The main result}

For the special projections given in Section 3.2, we will demonstrate the following lemma by the standard approximation theory [4].

Lemma 5.1. We choose the initial solution given in (4.19) then we have

$$
\left\|c_{f}(x, y, 0)-c_{h}(x, y, 0)\right\|+\left\|p(x, y, 0)-p_{h}(x, y, 0)\right\|+\left\|u(x, y, 0)-u_{h}(x, y, 0)\right\| \leq C h^{k+1} .
$$

Now we state the main theorem.

Theorem 5.2. Let $c_{f} \in L^{\infty}\left(0, T ; H^{k+3}\right), \mathbf{s} \in L^{\infty}\left(0, T ;\left(H^{k+2}\right)^{2}\right), \mathbf{u} \in L^{\infty}\left(0, T ;\left(H^{k+2}\right)^{2}\right), \phi \in L^{\infty}\left(0, T ; H^{k+3}\right)$ be the exact solutions of the problem (4.1)-(4.5), and let $c_{h}, \mathbf{s}_{\mathbf{h}}, \mathbf{u}_{\mathbf{h}}, \phi_{h}, p_{h}$ be the numerical solutions of IMEX$L D G(k, r)$ scheme $(r=1,2)$ with initial discretization given as (4.19). If the finite element space is the piecewise tensor product polynomials of degree at most $k$ and assume that for $r=1$,

$$
C \tau \leq \min \left(\frac{1}{2}, \frac{\gamma}{2}, \frac{\phi_{0}}{2}\right)
$$

for $r=2$,

$$
h^{2 k-3 \delta} \leq \tau \leq h^{\frac{2+3 \delta}{3}},
$$

where $\delta \leq \frac{3 k-1}{6}(k \geq 1)$ is given constant. Then we have the error estimate

$$
\left\|e_{c}^{n}\right\|^{2}+\left\|e_{p}^{n}\right\|^{2}+\left\|e_{\phi}^{n}\right\|^{2}+\tau \sum_{m=1}^{n}\left\|\mathbf{e}_{\mathbf{u}}^{\mathbf{m}}\right\|^{2}+\tau \sum_{m=1}^{n}\left\|\mathbf{e}_{\mathbf{s}}^{\mathbf{m}}\right\|^{2} \leq C\left(h^{2 k+2}+\tau^{2 r}\right), \quad \forall n \geq 1,
$$

for two IMEX-LDG schemes, provided $h$ is sufficiently small. 


\subsection{Proof of the main result}

In this paper, we use $e$ to denote the error between the exact and numerical solutions, i.e. $e_{c}=c_{f}-c_{h}, e_{p}=$ $p-p_{h}, \mathbf{e}_{\mathbf{u}}=\mathbf{u}-\mathbf{u}_{\mathbf{h}}, \mathbf{e}_{\mathbf{s}}=\mathbf{s}-\mathbf{s}_{\mathbf{h}}, e_{\phi}=\phi-\phi_{h}$. As the general treatment of the finite element methods, we split the errors into two terms as

$$
\begin{array}{lll}
e_{c}=\xi_{c}-\eta_{c}, & \eta_{c}=P^{+} c_{f}-c_{f}, & \xi_{c}=P^{+} c_{f}-c_{h}, \\
e_{p}=\xi_{p}-\eta_{p}, & \eta_{p}=P^{+} p-p, & \xi_{p}=P^{+} p-p_{h} \\
\mathbf{e}_{\mathbf{u}}=\boldsymbol{\xi}_{\boldsymbol{u}}-\boldsymbol{\eta}_{\boldsymbol{u}}, & \boldsymbol{\eta}_{\boldsymbol{u}}=\Pi^{-} \mathbf{u}-\mathbf{u}, & \boldsymbol{\xi}_{\boldsymbol{u}}=\Pi^{-} \mathbf{u}-\mathbf{u}_{\mathbf{h}}, \\
\mathbf{e}_{\mathbf{s}}=\boldsymbol{\xi}_{\boldsymbol{s}}-\boldsymbol{\eta}_{\boldsymbol{s}}, & \boldsymbol{\eta}_{\boldsymbol{s}}=\Pi^{-} \mathbf{s}-\mathbf{s}, & \boldsymbol{\xi}_{\boldsymbol{s}}=\Pi^{-} \mathbf{s}-\mathbf{s}_{\mathbf{h}}
\end{array}
$$

Based on the above notations, it is easy to verify that

$$
\mathcal{L}^{d}\left(\boldsymbol{\eta}_{\boldsymbol{u}}, v\right)=\mathcal{L}^{d}\left(\boldsymbol{\eta}_{\boldsymbol{s}}, v\right)=0, \quad \forall v \in Q^{k}(K) .
$$

From Lemma 3.2, we have the following approximation properties.

Lemma 5.3. The projection errors satisfy, for any $n \geq 0$, the following properties

$$
\begin{gathered}
\left\|\eta_{c}^{n}\right\|+\left\|\eta_{p}^{n}\right\|+\left\|\boldsymbol{\eta}_{\boldsymbol{u}}^{n}\right\|+\left\|\boldsymbol{\eta}_{\boldsymbol{s}}^{n}\right\| \leq C h^{k+1}, \\
\left\|\eta_{c}^{n+1}-\eta_{c}^{n}\right\|+\left\|\eta_{p}^{n+1}-\eta_{p}^{n}\right\| \leq C h^{k+1} \tau .
\end{gathered}
$$

Then we follow [26] and make a priori error estimate: if $h$ is small enough, there holds

$$
\left\|\mathbf{e}_{\mathbf{u}}^{\mathbf{m}}\right\| \leq h^{1+\delta}, \quad 0 \leq m \leq n,
$$

which further implies

$$
\left\|\mathbf{u}_{\mathbf{h}}^{\mathbf{m}}\right\|_{\infty} \leq C, \quad 0 \leq m \leq n,
$$

by Lemma 3.4 and hypothesis 3 , for any $n \geq 0$. We would like to verify this a priori assumption in Section 5.2.2.

Lemma 5.4. Assume the time step restriction $C \tau \leq \min \left(\frac{1}{2}, \frac{\gamma}{2}, \frac{\phi_{0}}{2}\right)$ for $r=1$ and the temporal-spatial condition $h^{2 k-3 \delta} \leq \tau \leq h^{\frac{2+3 \delta}{3}}$ for $r=2$ hold. Under the a priori estimate $(5.7)$, the IMEX-LDG(k,r) scheme $(r=1,2)$ satisfies

$$
\left\|\xi_{c}^{n}\right\|^{2}+\left\|\xi_{p}^{n}\right\|^{2}+\left\|e_{\phi}^{n}\right\|^{2}+\tau \sum_{m=1}^{n}\left\|\boldsymbol{\xi}_{\boldsymbol{u}}^{\boldsymbol{m}}\right\|^{2}+\tau \sum_{m=1}^{n}\left\|\boldsymbol{\xi}_{\boldsymbol{s}}^{\boldsymbol{m}}\right\|^{2} \leq C\left(h^{2 k+2}+\tau^{2 r}\right), \quad \forall n \geq 1,
$$

where $C$ is independent of $n$ and $h$.

\subsubsection{Proof of main result with $r=1$}

In this section, we consider the first-order time integration. The exact solutions satisfy the following variational forms

$$
\begin{aligned}
\left(\gamma \frac{p^{n+1}-p^{n}}{\tau}, \zeta\right)+\left(\frac{\phi^{n+1}-\phi^{n}}{\tau}, \zeta\right) & =\mathcal{L}^{d}\left(\mathbf{u}^{\mathbf{n}+\mathbf{1}}, \zeta\right)+\left(f^{n}, \zeta\right)+\left(\varsigma_{1}^{n}, \zeta\right) \\
\left(\frac{\mu}{\kappa\left(\phi^{n+1}\right)} \mathbf{u}^{\mathbf{n}+\mathbf{1}}, \boldsymbol{\theta}\right) & =\mathcal{D}\left(p^{n+1}, \boldsymbol{\theta}\right) \\
\left(\frac{\phi^{n+1} c_{f}^{n+1}-\phi^{n} c_{f}^{n}}{\tau}, v\right) & =\mathcal{L}^{d}\left(\mathbf{s}^{\mathbf{n}+\mathbf{1}}, v\right)-\left(A a_{v}\left(\phi^{n}\right) c_{f}^{n}, v\right)+\left(f_{p}^{n} c_{f}^{n}+f_{I}^{n} c_{I}^{n}, v\right)+\left(\varsigma_{2}^{n}, v\right) \\
\left(\left(\phi^{n+1} \mathbf{D}\left(\mathbf{u}^{\mathbf{n}+\mathbf{1}}\right)\right)^{-1} \mathbf{s}^{\mathbf{n}+\mathbf{1}}, \mathbf{w}\right) & =\left(\left(\phi^{n+1}\right)^{-1} \mathbf{M}_{\mathbf{e}}\left(\mathbf{u}^{\mathbf{n}+\mathbf{1}}\right) c_{f}^{n+1}, \mathbf{w}\right)+\mathcal{D}\left(c_{f}^{n+1}, \mathbf{w}\right)
\end{aligned}
$$




$$
\frac{\phi^{n+1}-\phi^{n}}{\tau}=B a_{v}\left(\phi^{n+1}\right) c_{f}^{n}+\varsigma_{3}^{n},
$$

where $\zeta, v \in W_{h}^{K}$ and $\boldsymbol{\theta}, \mathbf{w} \in \mathbf{W}_{\mathbf{h}}^{\mathbf{K}}$. Here $\varsigma_{i}^{n}, i=1,2,3$ are local truncation errors satisfying

$$
\left\|\varsigma_{i}^{n}\right\| \leq C \tau, \quad i=1,2,3, \quad \forall n \geq 0,
$$

under the assumption that the exact solutions are second-order differentiable. Subtracting (5.10)-(5.14) from (4.14)-(4.18), we get the following error equations

$$
\begin{aligned}
\left(\gamma \frac{e_{p}^{n+1}-e_{p}^{n}}{\tau}, \zeta\right)+\left(\frac{e_{\phi}^{n+1}-e_{\phi}^{n}}{\tau}, \zeta\right)= & \mathcal{L}^{d}\left(\mathbf{e}_{\mathbf{u}}^{\mathbf{n}+\mathbf{1}}, \zeta\right)+\left(\varsigma_{1}^{n}, \zeta\right) \\
\left(\frac{\mu}{\kappa\left(\phi^{n+1}\right)} \mathbf{u}^{\mathbf{n}+\mathbf{1}}-\frac{\mu}{\kappa\left(\phi_{h}^{n+1}\right)} \mathbf{u}_{\mathbf{h}}^{\mathbf{n}+\mathbf{1}}, \boldsymbol{\theta}\right)= & \mathcal{D}\left(e_{p}^{n+1}, \boldsymbol{\theta}\right) \\
\left(\frac{\phi^{n+1} c_{f}^{n+1}-\phi^{n} c_{f}^{n}-\left(\phi_{h}^{n+1} c_{h}^{n+1}-\phi_{h}^{n} c_{h}^{n}\right)}{\tau}, v\right)= & \mathcal{L}^{d}\left(\boldsymbol{e}_{s}^{n+\mathbf{1}}, v\right)-\left(A a_{v}\left(\phi^{n}\right) c_{f}^{n}-A a_{v}\left(\phi_{h}^{n}\right) c_{h}^{n}, v\right) \\
& +\left(f_{p}^{n} e_{c}^{n}, v\right)+\left(\varsigma_{2}^{n}, v\right), \\
\left(\left(\phi^{n+1} \mathbf{D}\left(\mathbf{u}^{\mathbf{n}+\mathbf{1}}\right)\right)^{-1} \mathbf{s}^{\mathbf{n}+\mathbf{1}}-\left(\phi_{h}^{n+1} \mathbf{D}\left(\mathbf{u}_{\mathbf{h}}^{\mathbf{n}+\mathbf{1}, \mathbf{M}}\right)\right)^{-1} \mathbf{s}_{\mathbf{h}}^{\mathbf{n}+\mathbf{1}}, \mathbf{w}\right)= & \left(\left(\phi^{n+1}\right)^{-1} \mathbf{M}_{\mathbf{e}}\left(\mathbf{u}^{\mathbf{n}+\mathbf{1}}\right) c_{f}^{n+1}-\left(\phi_{h}^{n+1}\right)^{-1}\right. \\
\frac{e_{\phi}^{n+1}-e_{\phi}^{n}}{\tau}= & \left.B \mathbf{M}_{\mathbf{e}}\left(\mathbf{u}_{\mathbf{h}}^{\mathbf{n}+\mathbf{1}}\right) c_{h}^{n+1}, \mathbf{w}\right)+\mathcal{D}\left(\phi_{c}^{n+1}\right) c_{f}^{n}-B a_{v}\left(\phi_{h}^{n+1}\right) \bar{c}_{h}^{n}+\varsigma_{3}^{n} .
\end{aligned}
$$

Proof. Now, we start to prove Lemma 5.4.

Step 1. Multiplying (5.20) by $e_{\phi}^{n+1}$ and integrating it over $\Omega$, we obtain

$$
\left(\frac{e_{\phi}^{n+1}-e_{\phi}^{n}}{\tau}, e_{\phi}^{n+1}\right)=\left(B a_{v}\left(\phi^{n+1}\right) c_{f}^{n}-B a_{v}\left(\phi_{h}^{n+1}\right) \bar{c}_{h}^{n}, e_{\phi}^{n+1}\right)+\left(\varsigma_{3}^{n}, e_{\phi}^{n+1}\right) .
$$

Noticing that

$$
B a_{v}\left(\phi^{n+1}\right) c_{f}^{n}-B a_{v}\left(\phi_{h}^{n+1}\right) \bar{c}_{h}^{n}=B\left(a_{v}\left(\phi^{n+1}\right)-a_{v}\left(\phi_{h}^{n+1}\right)\right) c_{f}^{n}+B a_{v}\left(\phi_{h}^{n+1}\right)\left(c_{f}^{n}-\bar{c}_{h}^{n}\right) .
$$

With the Lipschitz continuity of $a_{v}(\cdot)$ and the fact $\left|c_{f}^{n}-\bar{c}_{h}^{n}\right| \leq\left|c_{f}^{n}-c_{h}^{n}\right|$, we have

$$
\begin{aligned}
& \frac{1}{2}\left\|e_{\phi}^{n+1}\right\|^{2}+\frac{1}{2}\left\|e_{\phi}^{n+1}-e_{\phi}^{n}\right\|^{2}-\frac{1}{2}\left\|e_{\phi}^{n}\right\|^{2} \\
& \quad=\tau\left(B\left(a_{v}\left(\phi^{n+1}\right)-a_{v}\left(\phi_{h}^{n+1}\right)\right) c_{f}^{n}, e_{\phi}^{n+1}\right)+\tau\left(B a_{v}\left(\phi_{h}^{n+1}\right)\left(c_{f}^{n}-\bar{c}_{h}^{n}\right), e_{\phi}^{n+1}\right)+\tau\left(\varsigma_{3}^{n}, e_{\phi}^{n+1}\right) \\
& \quad \leq C \tau\left(\left\|e_{\phi}^{n+1}\right\|+\left\|\xi_{c}^{n}\right\|+\left\|\eta_{c}^{n}\right\|+\tau\right)\left\|e_{\phi}^{n+1}\right\| \\
& \quad \leq C \tau\left(\left\|e_{\phi}^{n}\right\|+\left\|e_{\phi}^{n+1}-e_{\phi}^{n}\right\|+\left\|\xi_{c}^{n}\right\|+\left\|\eta_{c}^{n}\right\|+\tau\right)\left(\left\|e_{\phi}^{n}\right\|+\left\|e_{\phi}^{n+1}-e_{\phi}^{n}\right\|\right) \\
& \quad \leq C \tau\left(\left\|e_{\phi}^{n}\right\|^{2}+\left\|e_{\phi}^{n+1}-e_{\phi}^{n}\right\|^{2}+\left\|\xi_{c}^{n}\right\|^{2}+h^{2 k+2}+\tau^{2}\right)
\end{aligned}
$$


where in the second step, we use hypotheses 2,3 and the boundedness of $\phi_{h}^{n+1}$ while the last step requires Lemma 5.3. Summing it over $n$, we get

$$
\frac{1}{2}\left\|e_{\phi}^{n+1}\right\|^{2}+\frac{1}{2} \sum_{m=0}^{n}\left\|e_{\phi}^{m+1}-e_{\phi}^{m}\right\|^{2} \leq C \tau \sum_{m=0}^{n}\left(\left\|e_{\phi}^{m}\right\|^{2}+\left\|\xi_{c}^{m}\right\|^{2}+\left\|e_{\phi}^{m+1}-e_{\phi}^{m}\right\|^{2}\right)+C\left(h^{2 k+2}+\tau^{2}\right) .
$$

On the other hand, it is observed that

$$
\frac{e_{\phi}^{n+1}-e_{\phi}^{n}}{\tau}=-\frac{R c_{f}^{n}}{1-\phi_{0}} e_{\phi}^{n+1}+\frac{R}{1-\phi_{0}}\left(1-\phi_{h}^{n+1}\right)\left(c_{f}^{n}-\bar{c}_{h}^{n}\right)+\varsigma_{3}^{n},
$$

leading to

$$
\begin{aligned}
\left\|\frac{e_{\phi}^{n+1}-e_{\phi}^{n}}{\tau}\right\| & \leq\left\|\frac{R c_{f}^{n}}{1-\phi_{0}} e_{\phi}^{n+1}\right\|+\left\|\frac{R}{1-\phi_{0}}\left(1-\phi_{h}^{n+1}\right)\left(c_{f}^{n}-\bar{c}_{h}^{n}\right)\right\|+C \tau \\
& \leq C\left(\left\|e_{\phi}^{n+1}\right\|+\left\|\xi_{c}^{n}\right\|+h^{k+1}+\tau\right) .
\end{aligned}
$$

Step 2. We take $\zeta=\xi_{p}^{n+1}$ in (5.16) and $\boldsymbol{\theta}=\boldsymbol{\xi}_{\boldsymbol{u}}^{\boldsymbol{n}+\boldsymbol{1}}$ in (5.17), and sum up these two equations to get

$$
\left(\gamma \frac{\xi_{p}^{n+1}-\xi_{p}^{n}}{\tau}, \xi_{p}^{n+1}\right)+\left(\frac{\mu}{\kappa\left(\phi_{h}^{n+1}\right)} \boldsymbol{\xi}_{\boldsymbol{u}}^{\boldsymbol{n}+\mathbf{1}}, \boldsymbol{\xi}_{\boldsymbol{u}}^{\boldsymbol{n}+\mathbf{1}}\right)=\sum_{i=1}^{4} T_{i}
$$

where

$$
\begin{aligned}
& T_{1}=\left(\gamma \frac{\eta_{p}^{n+1}-\eta_{p}^{n}}{\tau}, \xi_{p}^{n+1}\right)-\left(\frac{e_{\phi}^{n+1}-e_{\phi}^{n}}{\tau}, \xi_{p}^{n+1}\right) \\
& T_{2}=\left(\frac{\mu}{\kappa\left(\phi_{h}^{n+1}\right)} \boldsymbol{\eta}_{\boldsymbol{u}}^{\boldsymbol{n}+\mathbf{1}}, \boldsymbol{\xi}_{\boldsymbol{u}}^{\boldsymbol{n}+\mathbf{1}}\right)+\left(\left(\frac{\mu}{\kappa\left(\phi_{h}^{n+1}\right)}-\frac{\mu}{\kappa\left(\phi^{n+1}\right)}\right) \mathbf{u}^{\mathbf{n}+\mathbf{1}}, \boldsymbol{\xi}_{\boldsymbol{u}}^{\boldsymbol{n}+\mathbf{1}}\right) \\
& T_{3}=-\mathcal{D}\left(\eta_{p}^{n+1}, \boldsymbol{\xi}_{\boldsymbol{u}}^{\boldsymbol{n}+\mathbf{1}}\right), \\
& T_{4}=\left(\varsigma_{1}^{n}, \xi_{p}^{n+1}\right) .
\end{aligned}
$$

Now we estimate $T_{i}(i=1,2,3,4)$ term by term. Using Schwarz inequality, Lemma 5.3 and (5.24), we have

$$
T_{1} \leq C\left(\left\|e_{\phi}^{n+1}\right\|+\left\|\xi_{c}^{n}\right\|+h^{k+1}+\tau\right)\left\|\xi_{p}^{n+1}\right\| .
$$

Use hypothesis 3 and the Lipschitz continuity of $\kappa(\cdot)^{-1}$ to obtain

$$
T_{2} \leq C\left(h^{k+1}+\left\|e_{\phi}^{n+1}\right\|\right)\left\|\boldsymbol{\xi}_{u}^{n+1}\right\| .
$$

The estimate of $T_{3}$ follows from Lemma 3.3, then

$$
T_{3} \leq C h^{k+1}\left\|p^{n+1}\right\|_{k+2}\left\|\boldsymbol{\xi}_{u}^{n+1}\right\| .
$$

For $T_{4}$, we can use Schwarz inequality to get

$$
T_{4} \leq C \tau\left\|\xi_{p}^{n+1}\right\| .
$$

Substitute all the above estimates into (5.25), we have

$$
\frac{\gamma}{2}\left\|\xi_{p}^{n+1}\right\|^{2}+\frac{\gamma}{2}\left\|\xi_{p}^{n+1}-\xi_{p}^{n}\right\|^{2}-\frac{\gamma}{2}\left\|\xi_{p}^{n}\right\|^{2}+\tau\left\|\left(\frac{\mu}{\kappa\left(\phi_{h}^{n+1}\right)}\right)^{1 / 2} \xi_{u}^{n+1}\right\|^{2}
$$




$$
\begin{aligned}
\leq & C \tau\left(\left\|e_{\phi}^{n+1}\right\|+\left\|\xi_{c}^{n}\right\|+h^{k+1}+\tau\right)\left\|\xi_{p}^{n+1}\right\|+C \tau\left(\left\|e_{\phi}^{n+1}\right\|+h^{k+1}\right)\left\|\boldsymbol{\xi}_{u}^{n+1}\right\| \\
\leq & C \tau\left(\left\|e_{\phi}^{n}\right\|+\left\|e_{\phi}^{n+1}-e_{\phi}^{n}\right\|+\left\|\xi_{c}^{n}\right\|+h^{k+1}+\tau\right)\left(\left\|\xi_{p}^{n}\right\|+\left\|\xi_{p}^{n+1}-\xi_{p}^{n}\right\|\right) \\
& +C \tau\left(\left\|e_{\phi}^{n}\right\|+\left\|e_{\phi}^{n+1}-e_{\phi}^{n}\right\|+h^{k+1}\right)\left\|\xi_{u}^{n+1}\right\| \\
\leq & C \tau\left(\left\|e_{\phi}^{n}\right\|^{2}+\left\|e_{\phi}^{n+1}-e_{\phi}^{n}\right\|^{2}+\left\|\xi_{c}^{n}\right\|^{2}+\left\|\xi_{p}^{n}\right\|^{2}+\left\|\xi_{p}^{n+1}-\xi_{p}^{n}\right\|^{2}+h^{2 k+2}+\tau^{2}\right) \\
& +\varepsilon \tau\left\|\left(\frac{\mu}{\kappa\left(\phi_{h}^{n+1}\right)}\right)^{-1 / 2} \boldsymbol{\xi}_{u}^{n+1}\right\|^{2},
\end{aligned}
$$

where the last step follows from Young's inequality. Taking $\varepsilon=\frac{1}{8}$, then we sum it over $n$ to obtain

$$
\begin{aligned}
& \frac{\gamma}{2}\left\|\xi_{p}^{n+1}\right\|^{2}+\frac{\gamma}{2} \sum_{m=0}^{n}\left\|\xi_{p}^{m+1}-\xi_{p}^{m}\right\|^{2}+\frac{7 \tau}{8} \sum_{m=0}^{n}\left\|\left(\frac{\mu}{\kappa\left(\phi_{h}^{m+1}\right)}\right)^{1 / 2} \boldsymbol{\xi}_{u}^{m+1}\right\|^{2} \\
& \quad \leq C \tau \sum_{m=0}^{n}\left(\left\|e_{\phi}^{m}\right\|^{2}+\left\|\xi_{c}^{m}\right\|^{2}+\left\|\xi_{p}^{m}\right\|^{2}+\left\|e_{\phi}^{m+1}-e_{\phi}^{m}\right\|^{2}+\left\|\xi_{p}^{m+1}-\xi_{p}^{m}\right\|^{2}\right)+C\left(h^{2 k+2}+\tau^{2}\right) .
\end{aligned}
$$

Step 3. Taking $v=\xi_{c}^{n+1}$ in (5.18) and $\mathbf{w}=\boldsymbol{\xi}_{\boldsymbol{s}}^{\boldsymbol{n}+\mathbf{1}}$ in (5.19), and then summing these two equations, we get

$$
\left(\frac{\left(\xi_{c}^{n+1}-\xi_{c}^{n}\right) \phi_{h}^{n+1}}{\tau}, \xi_{c}^{n+1}\right)+\left(\left(\phi_{h}^{n+1} \mathbf{D}\left(\mathbf{u}_{\mathbf{h}}^{\mathbf{n}+\mathbf{1}, \mathbf{M}}\right)\right)^{-1} \boldsymbol{\xi}_{s}^{n+1}, \boldsymbol{\xi}_{s}^{n+\mathbf{1}}\right)=\sum_{i=1}^{6} R_{i}
$$

where

$$
\begin{aligned}
R_{1}= & \left(\frac{\left(\eta_{c}^{n+1}-\eta_{c}^{n}\right) \phi_{h}^{n+1}}{\tau}, \xi_{c}^{n+1}\right)-\left(\frac{\left(c_{f}^{n+1}-c_{f}^{n}\right) e_{\phi}^{n+1}}{\tau}, \xi_{c}^{n+1}\right)-\left(\frac{\left(e_{\phi}^{n+1}-e_{\phi}^{n}\right) c_{f}^{n}}{\tau}, \xi_{c}^{n+1}\right) \\
& -\left(\frac{\left(\phi_{h}^{n+1}-\phi_{h}^{n}\right) \xi_{c}^{n}}{\tau}, \xi_{c}^{n+1}\right)+\left(\frac{\left(\phi_{h}^{n+1}-\phi_{h}^{n}\right) \eta_{c}^{n}}{\tau}, \xi_{c}^{n+1}\right), \\
R_{2}= & \left(\left(\left(\phi_{h}^{n+1} \mathbf{D}\left(\mathbf{u}_{\mathbf{h}}^{\mathbf{n}+\mathbf{1}, \mathbf{M}}\right)\right)^{-1}-\left(\phi^{n+1} \mathbf{D}\left(\mathbf{u}^{\mathbf{n}+\mathbf{1}}\right)\right)^{-1}\right) \mathbf{s}^{\mathbf{n}+\mathbf{1}}, \boldsymbol{\xi}_{s}^{n+\mathbf{1}}\right) \\
& +\left(\left(\left(\phi^{n+1}\right)^{-1} \mathbf{M}_{\mathbf{e}}\left(\mathbf{u}^{\mathbf{n}+\mathbf{1}}\right)-\left(\phi_{h}^{n+1}\right)^{-1} \mathbf{M}_{\mathbf{e}}\left(\mathbf{u}_{\mathbf{h}}^{\mathbf{n}+\mathbf{1}}\right)\right) c_{f}^{n+1}, \boldsymbol{\xi}_{s}^{n+\mathbf{1}}\right), \\
R_{3}= & -\mathcal{D}\left(\eta_{c}^{n+1}, \boldsymbol{\xi}_{s}^{n+\mathbf{1}}\right), \\
R_{4}= & \left(\left(\phi_{h}^{n+1} \mathbf{D}\left(\mathbf{u}_{\mathbf{h}}^{\mathbf{n}+\mathbf{1}, \mathbf{M}}\right)\right)^{-1} \boldsymbol{\eta}_{s}^{n+\mathbf{1}}, \boldsymbol{\xi}_{s}^{n+\mathbf{1}}\right)+\left(\left(\phi_{h}^{n+1}\right)^{-1} \mathbf{M}_{\mathbf{e}}\left(\mathbf{u}_{\mathbf{h}}^{\mathbf{n}+\mathbf{1}}\right) e_{c}^{n+1}, \boldsymbol{\xi}_{s}^{n+\mathbf{1}}\right), \\
R_{5}= & \left(f_{p}^{n} e_{c}^{n}, \xi_{c}^{n+1}\right)+\left(\varsigma_{2}^{n}, \xi_{c}^{n+1}\right), \\
R_{6}= & A\left(a_{v}\left(\phi_{h}^{n}\right) c_{h}^{n}-a_{v}\left(\phi^{n}\right) c_{f}^{n}, \xi_{c}^{n+1}\right) .
\end{aligned}
$$

Now we estimate $R_{i}^{\prime} s$ term by term. Using Theorem 4.4, Lemma 5.3, hypothesis 3 and (5.24), we have

$$
R_{1} \leq C\left(h^{k+1}+\left\|e_{\phi}^{n+1}\right\|+\left\|\xi_{c}^{n}\right\|+\tau\right)\left\|\xi_{c}^{n+1}\right\| .
$$


Following [11], we take $S>2\|\mathbf{u}\|_{\infty}$ in the cut-off operator in (4.7) to obtain $\left|\mathbf{u}^{\mathbf{n}+\mathbf{1}}-\mathbf{u}_{\mathbf{h}}^{\mathbf{n}+\mathbf{1}, \mathbf{M}}\right| \leq$ $\left|\mathbf{u}^{\mathbf{n}+\mathbf{1}}-\mathbf{u}_{\mathbf{h}}^{\mathbf{n}+\mathbf{1}}\right|$, which further yields

$$
\begin{aligned}
R_{2}= & \left(\left(\left(\phi_{h}^{n+1} \mathbf{D}\left(\mathbf{u}_{\mathbf{h}}^{\mathbf{n}+\mathbf{1}, \mathbf{M}}\right)\right)^{-1}-\left(\phi^{n+1} \mathbf{D}\left(\mathbf{u}_{\mathbf{h}}^{\mathbf{n}+\mathbf{1}, \mathbf{M}}\right)\right)^{-1}\right) \mathbf{s}^{\mathbf{n}+\mathbf{1}}, \boldsymbol{\xi}_{s}^{n+\mathbf{1}}\right) \\
& +\left(\left(\left(\phi^{n+1} \mathbf{D}\left(\mathbf{u}_{\mathbf{h}}^{\mathbf{n}+\mathbf{1}, \mathbf{M}}\right)\right)^{-1}-\left(\phi^{n+1} \mathbf{D}\left(\mathbf{u}^{\mathbf{n}+\mathbf{1}}\right)\right)^{-1}\right) \mathbf{s}^{\mathbf{n}+\mathbf{1}}, \boldsymbol{\xi}_{s}^{n+\mathbf{1}}\right) \\
& +\left(\left(\left(\phi^{n+1}\right)^{-1} \mathbf{M}_{\mathbf{e}}\left(\mathbf{u}^{\mathbf{n}+\mathbf{1}}\right)-\left(\phi^{n+1}\right)^{-1} \mathbf{M}_{\mathbf{e}}\left(\mathbf{u}_{\mathbf{h}}^{\mathbf{n}+\mathbf{1}}\right)\right) c_{f}^{n+1}, \boldsymbol{\xi}_{s}^{n+\mathbf{1}}\right) \\
& +\left(\left(\left(\phi^{n+1}\right)^{-1} \mathbf{M}_{\mathbf{e}}\left(\mathbf{u}_{\mathbf{h}}^{\mathbf{n}+\mathbf{1}}\right)-\left(\phi_{h}^{n+1}\right)^{-1} \mathbf{M}_{\mathbf{e}}\left(\mathbf{u}_{\mathbf{h}}^{\mathbf{n}+\mathbf{1}}\right)\right) c_{f}^{n+1}, \boldsymbol{\xi}_{s}^{n+\mathbf{1}}\right) \\
\leq & C\left(\left\|\boldsymbol{\xi}_{u}^{n+\mathbf{1}}\right\|+\left\|e_{\phi}^{n+1}\right\|+h^{k+1}\right)\left\|\boldsymbol{\xi}_{s}^{n+\mathbf{1}}\right\|
\end{aligned}
$$

by hypothesis 3 , the boundedness of $\mathbf{D}(\cdot)^{-\mathbf{1}}$ in (4.26) and the Lipschitz continuity of $\mathbf{D}(\cdot)^{-\mathbf{1}}$ and $\mathbf{M}_{\mathbf{e}}(\cdot)$ given in (4.28) and (4.30) respectively. Here $C$ depends on $\left\|c_{f}^{n+1}\right\|_{\infty}$ and $\left\|\mathbf{s}^{\mathbf{n}+\mathbf{1}}\right\|_{\infty}$. The estimate of $R_{3}$ requires hypothesis 3 and Lemma 3.3,

$$
R_{3} \leq C h^{k+1}\left\|c_{f}\right\|_{k+2}\left\|\boldsymbol{\xi}_{s}^{n+1}\right\| .
$$

Using the boundedness of $\phi_{h}^{n+1}, \mathbf{D}\left(\mathbf{u}_{\mathbf{h}}^{\mathbf{n}+\mathbf{1}, \mathbf{M}}\right)$ and $\mathbf{M}_{\mathbf{e}}\left(\mathbf{u}_{\mathbf{h}}^{\mathbf{n}+\mathbf{1}}\right)$, we have

$$
R_{4} \leq C\left(\left\|\xi_{c}^{n+1}\right\|+h^{k+1}\right)\left\|\boldsymbol{\xi}_{s}^{n+1}\right\| .
$$

The estimate of $R_{5}$ follows from Schwarz inequality

$$
R_{5} \leq C\left(\left\|\xi_{c}^{n}\right\|+h^{k+1}+\tau\right)\left\|\xi_{c}^{n+1}\right\| .
$$

Finally, we estimate $R_{6}$. By using hypothesis 2,3 and Lemma 2.1, we have

$$
\begin{aligned}
R_{6} & =-\left(A\left(a_{v}\left(\phi^{n}\right) c_{f}^{n}-a_{v}\left(\phi_{h}^{n}\right) c_{f}^{n}+a_{v}\left(\phi_{h}^{n}\right) c_{f}^{n}-a_{v}\left(\phi_{h}^{n}\right) c_{h}^{n}\right), \xi_{c}^{n+1}\right) \\
& =-\left(A c_{f}^{n}\left(a_{v}\left(\phi^{n}\right)-a_{v}\left(\phi_{h}^{n}\right)\right)+A a_{v}\left(\phi_{h}^{n}\right)\left(c_{f}^{n}-c_{h}^{n}\right), \xi_{c}^{n+1}\right) \\
& \leq C\left(\left\|e_{\phi}^{n}\right\|+\left\|\xi_{c}^{n}\right\|+h^{k+1}\right)\left\|\xi_{c}^{n+1}\right\| .
\end{aligned}
$$

Substituting all the above estimates into (5.28) and using $\phi_{h}^{n+1}=\phi_{h}^{n}+\tau B a_{v}\left(\phi_{h}^{n+1}\right) \bar{c}_{h}^{n}$ again, we get

$$
\begin{aligned}
\frac{1}{2} \| & \sqrt{\phi_{h}^{n+1}} \xi_{c}^{n+1}\left\|^{2}+\frac{1}{2}\right\| \sqrt{\phi_{h}^{n+1}}\left(\xi_{c}^{n+1}-\xi_{c}^{n}\right)\left\|^{2}-\frac{1}{2}\right\| \sqrt{\phi_{h}^{n}} \xi_{c}^{n}\left\|^{2}+\tau\right\|\left(\phi_{h}^{n+1} \mathbf{D}\left(\mathbf{u}_{\mathbf{h}}^{\mathbf{n}+\mathbf{1}, \mathbf{M}}\right)\right)^{-1 / 2} \boldsymbol{\xi}_{s}^{n+\mathbf{1}} \|^{2} \\
\leq & C \tau\left(h^{k+1}+\left\|e_{\phi}^{n+1}\right\|+\left\|e_{\phi}^{n}\right\|+\left\|\xi_{c}^{n}\right\|+\tau\right)\left\|\xi_{c}^{n+1}\right\| \\
& +C \tau\left(\left\|\boldsymbol{\xi}_{u}^{n+\mathbf{1}}\right\|+\left\|\xi_{c}^{n+1}\right\|+\left\|e_{\phi}^{n+1}\right\|+h^{k+1}\right)\left\|\boldsymbol{\xi}_{s}^{n+\mathbf{1}}\right\|+\frac{1}{2} \tau R \frac{1-\phi_{h}^{n+1}}{1-\phi_{0}}\left(\xi_{c}^{n}, \xi_{c}^{n}\right) \\
\leq & C \tau\left(\left\|e_{\phi}^{n}\right\|^{2}+\left\|e_{\phi}^{n+1}-e_{\phi}^{n}\right\|^{2}+\left\|\xi_{c}^{n}\right\|^{2}+\left\|\xi_{c}^{n+1}-\xi_{c}^{n}\right\|^{2}+\left\|\boldsymbol{\xi}_{\boldsymbol{u}}^{n+\mathbf{1}}\right\|^{2}+h^{2 k+2}+\tau^{2}\right) \\
& +\varepsilon \tau\left\|\left(\phi_{h}^{n+1} \mathbf{D}\left(\mathbf{u}_{\mathbf{h}}^{\mathbf{n}+\mathbf{1}, \mathbf{M}}\right)\right)^{-1 / 2} \boldsymbol{\xi}_{s}^{n+\mathbf{1}}\right\|^{2}
\end{aligned}
$$

where the last step follows from Young's inequality. Summing it over $n$, taking $\varepsilon=\frac{1}{8}$ and under the condition $C \tau \leq \min \left(\frac{1}{2}, \frac{\gamma}{2}, \frac{\phi_{0}}{2}\right)$, we have

$$
\frac{1}{2}\left\|\sqrt{\phi_{h}^{n+1}} \xi_{c}^{n+1}\right\|^{2}+\frac{7 \tau}{8} \sum_{m=0}^{n}\left\|\left(\phi_{h}^{m+1} \mathbf{D}\left(\mathbf{u}_{\mathbf{h}}^{\mathbf{m}+\mathbf{1}, \mathbf{M}}\right)\right)^{-1 / 2} \boldsymbol{\xi}_{s}^{m+\mathbf{1}}\right\|^{2}
$$




$$
\leq C \tau \sum_{m=0}^{n}\left(\left\|e_{\phi}^{m+1}-e_{\phi}^{m}\right\|^{2}+\left\|e_{\phi}^{m}\right\|^{2}+\left\|\xi_{c}^{m}\right\|^{2}+\left\|\xi_{p}^{m+1}-\xi_{p}^{m}\right\|^{2}+\left\|\xi_{p}^{m}\right\|^{2}\right)+C\left(h^{2 k+2}+\tau^{2}\right),
$$

and here we need to use (5.27). According to the initial discretization in (4.19), we have

$$
\begin{aligned}
& \xi_{c}^{0}=P^{+} c_{0}-c_{h}^{0}=P^{+} c_{0}-P^{+} c_{0}=0, \quad \xi_{p}^{0}=P^{+} p_{0}-p_{h}^{0}=P^{+} p_{0}-P^{+} p_{0}=0, \\
& e_{\phi}^{0}=\phi_{0}-\phi_{h}^{0}=\phi_{0}-\phi_{0}=0 .
\end{aligned}
$$

Combining (5.23), (5.27) and (5.30), and under the condition $C \tau \leq \min \left(\frac{1}{2}, \frac{\gamma}{2}, \frac{\phi_{0}}{2}\right)$, we get

$$
\begin{aligned}
& \frac{1}{2}\left\|e_{\phi}^{n+1}\right\|^{2}+\frac{\gamma}{2}\left\|\xi_{p}^{n+1}\right\|^{2}+\frac{1}{2}\left\|\sqrt{\phi_{h}^{n+1}} \xi_{c}^{n+1}\right\|^{2}+\frac{7 \tau}{8} \sum_{m=0}^{n}\left\|\left(\phi_{h}^{m+1} \mathbf{D}\left(\mathbf{u}_{\mathbf{h}}^{\mathbf{m}+\mathbf{1}, \mathbf{M}}\right)\right)^{-1 / 2} \boldsymbol{\xi}_{\boldsymbol{s}}^{\boldsymbol{m}+\mathbf{1}}\right\|^{2} \\
& \quad+\frac{7 \tau}{8} \sum_{m=0}^{n}\left\|\left(\frac{\mu}{\kappa\left(\phi_{h}^{m+1}\right)}\right)^{1 / 2} \boldsymbol{\xi}_{\boldsymbol{u}}^{m+1}\right\|^{2} \leq C \tau \sum_{m=0}^{n}\left(\left\|e_{\phi}^{m}\right\|^{2}+\left\|\xi_{c}^{m}\right\|^{2}+\left\|\xi_{p}^{m}\right\|^{2}\right)+C\left(h^{2 k+2}+\tau^{2}\right) .
\end{aligned}
$$

We can obtain Lemma 5.4 by applying Lemma 3.5 to (5.31).

\subsubsection{Proof of main result with $r=2$}

The proof is similar as but a little complicated than that in the previous section. The exact solutions satisfy, for any $n \geq 1$, the following forms

$$
\begin{aligned}
\left(\gamma \frac{p^{n+1}-p^{n}}{\tau}, \zeta\right)+\left(\frac{\phi^{n+1}-\phi^{n}}{\tau}, \zeta\right)= & \frac{3}{4} \mathcal{L}^{d}\left(\mathbf{u}^{\mathbf{n}+\mathbf{1}}, \zeta\right)+\frac{1}{4} \mathcal{L}^{d}\left(\mathbf{u}^{\mathbf{n}-\mathbf{1}}, \zeta\right) \\
& +\frac{3}{2}\left(f^{n}, \zeta\right)-\frac{1}{2}\left(f^{n-1}, \zeta\right)+\left(\varsigma_{1}^{n}, \zeta\right) \\
\left(\frac{\mu}{\kappa\left(\phi^{n+1}\right)} \mathbf{u}^{\mathbf{n}+\mathbf{1}}, \boldsymbol{\theta}\right)= & \mathcal{D}\left(p^{n+1}, \boldsymbol{\theta}\right), \\
\left(\frac{\phi^{n+1} c_{f}^{n+1}-\phi^{n} c_{f}^{n}}{\tau}, v\right)= & \frac{3}{4} \mathcal{L}^{d}\left(\mathbf{s}^{\mathbf{n}+\mathbf{1}}, v\right)+\frac{1}{4} \mathcal{L}^{d}\left(\mathbf{s}^{\mathbf{n}-\mathbf{1}}, v\right)-\frac{3}{2}\left(A a_{v}\left(\phi^{n}\right) c_{f}^{n}, v\right) \\
& +\frac{1}{2}\left(A a_{v}\left(\phi^{n-1}\right) c_{f}^{n-1}, v\right)+\frac{3}{2}\left(f_{p}^{n} c_{f}^{n}+f_{I}^{n} c_{I}^{n}, v\right) \\
& -\frac{1}{2}\left(f_{p}^{n-1} c_{f}^{n-1}+f_{I}^{n-1} c_{I}^{n-1}, v\right)+\left(\varsigma_{2}^{n}, v\right), \\
\left(\left(\phi^{n+1} \mathbf{D}\left(\mathbf{u}^{\mathbf{n}+\mathbf{1}}\right)\right)^{-1} \mathbf{s}^{\mathbf{n}+\mathbf{1}}, \mathbf{w}\right)= & \left(\left(\phi^{n+1}\right)^{-1} \mathbf{M}_{\mathbf{e}}\left(\mathbf{u}^{\mathbf{n}+\mathbf{1}}\right) c_{f}^{n+1}, \mathbf{w}\right)+\mathcal{D}\left(c_{f}^{n+1}, \mathbf{w}\right), \\
\frac{\phi^{n+1}-\phi^{n}}{\tau}= & \frac{1}{2} B a_{v}\left(\phi^{n+1}\right) \bar{c}_{f}^{n+1, *}+\frac{1}{2} B a_{v}\left(\phi^{n}\right) \bar{c}_{f}^{n}+\varsigma_{3}^{n} .
\end{aligned}
$$

Here $\varsigma_{i}^{n},(i=1,2,3)$ is local truncation error which satisfies

$$
\left\|\varsigma_{i}^{n}\right\| \leq C \tau^{2}, \quad n \geq 1
$$

under the assumption that the exact solutions are third-order differentiable. Then we can get the error equations.

$$
\left(\gamma \frac{e_{p}^{n+1}-e_{p}^{n}}{\tau}, \zeta\right)+\left(\frac{e_{\phi}^{n+1}-e_{\phi}^{n}}{\tau}, \zeta\right)=\frac{3}{4} \mathcal{L}^{d}\left(\mathbf{e}_{\mathbf{u}}^{\mathbf{n}+\mathbf{1}}, \zeta\right)+\frac{1}{4} \mathcal{L}^{d}\left(\mathbf{e}_{\mathbf{u}}^{\mathbf{n}-\mathbf{1}}, \zeta\right)+\left(\varsigma_{1}^{n}, \zeta\right),
$$




$$
\begin{aligned}
& \left(\frac{\mu}{\kappa\left(\phi^{n+1}\right)} \mathbf{u}^{\mathbf{n}+\mathbf{1}}-\frac{\mu}{\kappa\left(\phi_{h}^{n+1}\right)} \mathbf{u}_{\mathbf{h}}^{\mathbf{n}+\mathbf{1}}, \boldsymbol{\theta}\right)=\mathcal{D}\left(e_{p}^{n+1}, \boldsymbol{\theta}\right) \\
& \left(\frac{\phi^{n+1} c_{f}^{n+1}-\phi^{n} c_{f}^{n}-\left(\phi_{h}^{n+1} c_{h}^{n+1}-\phi_{h}^{n} c_{h}^{n}\right)}{\tau}, v\right)=\frac{3}{4} \mathcal{L}^{d}\left(\mathbf{e}_{\mathbf{s}}^{\mathbf{n}+\mathbf{1}}, v\right)+\frac{1}{4} \mathcal{L}^{d}\left(\mathbf{e}_{\mathbf{s}}^{\mathbf{n}-\mathbf{1}}, v\right) \\
& -\frac{3}{2}\left(A\left(a_{v}\left(\phi^{n}\right) c_{f}^{n}-a_{v}\left(\phi_{h}^{n}\right) c_{h}^{n}\right), v\right)+\frac{1}{2}\left(A\left(a_{v}\left(\phi^{n-1}\right) c_{f}^{n-1}-a_{v}\left(\phi_{h}^{n-1}\right) c_{h}^{n-1}\right), v\right) \\
& +\frac{3}{2}\left(f_{p}^{n} e_{c}^{n}, v\right)-\frac{1}{2}\left(f_{p}^{n-1} e_{c}^{n-1}, v\right)+\left(\varsigma_{2}^{n}, v\right) \\
& \left(\left(\phi^{n+1} \mathbf{D}\left(\mathbf{u}^{\mathbf{n}+\mathbf{1}}\right)\right)^{-1} \mathbf{s}^{\mathbf{n}+\mathbf{1}}-\left(\phi_{h}^{n+1} \mathbf{D}\left(\mathbf{u}_{\mathbf{h}}^{\mathbf{n}+\mathbf{1}, \mathbf{M}}\right)\right)^{-1} \mathbf{s}_{\mathbf{h}}^{\mathbf{n}+\mathbf{1}}, \mathbf{w}\right) \\
& =\left(\left(\phi^{n+1}\right)^{-1} \mathbf{M}_{\mathbf{e}}\left(\mathbf{u}^{\mathbf{n}+\mathbf{1}}\right) c_{f}^{n+1}-\left(\phi_{h}^{n+1}\right)^{-1} \mathbf{M}_{\mathbf{e}}\left(\mathbf{u}_{\mathbf{h}}^{\mathbf{n}+\mathbf{1}}\right) c_{h}^{n+1}, \mathbf{w}\right)+\mathcal{D}\left(e_{c}^{n+1}, \mathbf{w}\right), \\
& \frac{e_{\phi}^{n+1}-e_{\phi}^{n}}{\tau}=\frac{1}{2} B\left(a_{v}\left(\phi^{n+1}\right) c_{f}^{n+1, *}-a_{v}\left(\phi_{h}^{n+1}\right) \bar{c}_{h}^{n+1, *}\right)+\frac{1}{2} B\left(a_{v}\left(\phi^{n}\right) c_{f}^{n}-a_{v}\left(\phi_{h}^{n}\right) \bar{c}_{h}^{n}\right)+\varsigma_{3}^{n},
\end{aligned}
$$

for $n \geq 1$, and (5.16)-(5.20) for $n=0$, with

$$
\left\|\varsigma_{i}^{0}\right\| \leq C \tau, \quad i=1,2,3
$$

Proof. Now we start to prove Lemma 5.4 for $r=2$.

Step 1. Multiplying (5.42) with $e_{\phi}^{n+1}$, and integrating it over $\Omega$, we obtain

$$
\begin{aligned}
\left(e_{\phi}^{n+1}-e_{\phi}^{n}, e_{\phi}^{n+1}\right)= & \frac{\tau}{2}\left(B\left(a_{v}\left(\phi^{n+1}\right)-a_{v}\left(\phi_{h}^{n+1}\right)\right) c_{f}^{n+1, *}, e_{\phi}^{n+1}\right) \\
& +\frac{\tau}{2}\left(B a_{v}\left(\phi_{h}^{n+1}\right)\left(c_{f}^{n+1, *}-\bar{c}_{h}^{n+1, *}\right), e_{\phi}^{n+1}\right)+\frac{\tau}{2}\left(B a_{v}\left(\phi_{h}^{n}\right)\left(c_{f}^{n}-\bar{c}_{h}^{n}\right), e_{\phi}^{n+1}\right) \\
& +\frac{\tau}{2}\left(B\left(a_{v}\left(\phi^{n}\right)-a_{v}\left(\phi_{h}^{n}\right)\right) c_{f}^{n}, e_{\phi}^{n+1}\right)+\tau\left(\varsigma_{3}^{n}, e_{\phi}^{n+1}\right) .
\end{aligned}
$$

We use the fact $\left|c_{f}^{n}-\bar{c}_{h}^{n}\right| \leq\left|c_{f}^{n}-c_{h}^{n}\right|$ and the Lipschitz continuity of $a_{v}(\cdot)$ to get

$$
\begin{aligned}
\frac{1}{2}\left\|e_{\phi}^{n+1}\right\|^{2} & +\frac{1}{2}\left\|e_{\phi}^{n+1}-e_{\phi}^{n}\right\|^{2}-\frac{1}{2}\left\|e_{\phi}^{n}\right\|^{2} \\
& \leq C \tau\left(\left\|e_{\phi}^{n+1}\right\|+\left\|e_{\phi}^{n}\right\|+\left\|\xi_{c}^{n}\right\|+\left\|\xi_{c}^{n-1}\right\|+h^{k+1}+\tau^{2}\right)\left\|e_{\phi}^{n+1}\right\| \\
& \leq C \tau\left(\left\|e_{\phi}^{n+1}-e_{\phi}^{n}\right\|+\left\|e_{\phi}^{n}\right\|+\left\|\xi_{c}^{n}\right\|+\left\|\xi_{c}^{n-1}\right\|+h^{k+1}+\tau^{2}\right)\left(\left\|e_{\phi}^{n+1}-e_{\phi}^{n}\right\|+\left\|e_{\phi}^{n}\right\|\right) \\
& \leq C \tau\left(\left\|e_{\phi}^{n+1}-e_{\phi}^{n}\right\|^{2}+\left\|e_{\phi}^{n}\right\|^{2}+\left\|\xi_{c}^{n}\right\|^{2}+\left\|\xi_{c}^{n-1}\right\|^{2}+h^{2 k+2}+\tau^{4}\right) .
\end{aligned}
$$

Summing the above equation over $n$, we get

$$
\begin{aligned}
\frac{1}{2}\left\|e_{\phi}^{n+1}\right\|^{2}+\frac{1}{2} \sum_{m=1}^{n}\left\|e_{\phi}^{m+1}-e_{\phi}^{m}\right\|^{2} \leq & C \tau \sum_{m=1}^{n}\left(\left\|e_{\phi}^{m+1}-e_{\phi}^{m}\right\|^{2}+\left\|e_{\phi}^{m}\right\|^{2}+\left\|\xi_{c}^{m}\right\|^{2}+\left\|\xi_{c}^{m-1}\right\|^{2}\right) \\
& +C\left(h^{2 k+2}+\tau^{4}\right)+\frac{1}{2}\left\|e_{\phi}^{1}\right\|^{2} .
\end{aligned}
$$

Similarly, we have

$$
\left\|\frac{e_{\phi}^{n+1}-e_{\phi}^{n}}{\tau}\right\| \leq \frac{1}{2}\left\|\frac{R c_{f}^{n+1, *}}{1-\phi_{0}} e_{\phi}^{n+1}\right\|+\frac{1}{2}\left\|\frac{R}{1-\phi_{0}}\left(1-\phi_{h}^{n+1}\right)\left(c_{f}^{n+1, *}-\bar{c}_{h}^{n+1, *}\right)\right\|
$$




$$
\begin{aligned}
& +\frac{1}{2}\left\|\frac{R c_{f}^{n}}{1-\phi_{0}} e_{\phi}^{n}\right\|+\frac{1}{2}\left\|\frac{R}{1-\phi_{0}}\left(1-\phi_{h}^{n}\right)\left(c_{f}^{n}-\bar{c}_{h}^{n}\right)\right\|+C \tau^{2} \\
\leq & C\left(\left\|e_{\phi}^{n+1}\right\|+\left\|e_{\phi}^{n}\right\|+\left\|\xi_{c}^{n}\right\|+\left\|\xi_{c}^{n-1}\right\|+h^{k+1}+\tau^{2}\right) .
\end{aligned}
$$

Step 2. Taking $\xi_{p}^{n+1}$ in (5.38) and $\frac{3}{4} \boldsymbol{\xi}_{\boldsymbol{u}}^{\boldsymbol{n}+\boldsymbol{1}}$ in (5.39) and summing up to obtain

$$
\left(\gamma \frac{\xi_{p}^{n+1}-\xi_{p}^{n}}{\tau}, \xi_{p}^{n+1}\right)+\frac{3}{4}\left(\frac{\mu}{\kappa\left(\phi^{n+1}\right)} \boldsymbol{\xi}_{u}^{n+1}, \boldsymbol{\xi}_{\boldsymbol{u}}^{\boldsymbol{n}+\mathbf{1}}\right)=\sum_{i=1}^{5} T_{i}
$$

where

$$
\begin{aligned}
& T_{1}=\left(\gamma \frac{\eta_{p}^{n+1}-\eta_{p}^{n}}{\tau}, \xi_{p}^{n+1}\right)-\left(\frac{e_{\phi}^{n+1}-e_{\phi}^{n}}{\tau}, \xi_{p}^{n+1}\right) \\
& T_{2}=\frac{3}{4}\left(\frac{\mu}{\kappa\left(\phi^{n+1}\right)} \boldsymbol{\eta}_{\boldsymbol{u}}^{n+1}, \boldsymbol{\xi}_{u}^{n+1}\right)+\frac{3}{4}\left(\left(\frac{\mu}{\kappa\left(\phi_{h}^{n+1}\right)}-\frac{\mu}{\kappa\left(\phi^{n+1}\right)}\right) \mathbf{u}_{\mathbf{h}}^{\mathbf{n + 1}}, \boldsymbol{\xi}_{\boldsymbol{u}}^{n+\mathbf{1}}\right), \\
& T_{3}=\frac{1}{4} \mathcal{L}^{d}\left(\boldsymbol{\xi}_{u}^{n-\mathbf{1}}, \xi_{p}^{n+1}\right), \\
& T_{4}=-\frac{3}{4} \mathcal{D}\left(\eta_{p}^{n+1}, \boldsymbol{\xi}_{\boldsymbol{u}}^{n+\mathbf{1}}\right) \\
& T_{5}=\left(\varsigma_{1}^{n}, \xi_{p}^{n+1}\right) .
\end{aligned}
$$

Now we estimate $T_{i}^{\prime} s$ term by term. By Lemma 5.3 and (5.47), we get

$$
T_{1} \leq C\left(\left\|e_{\phi}^{n+1}\right\|+\left\|e_{\phi}^{n}\right\|+\left\|\xi_{c}^{n}\right\|+\left\|\xi_{c}^{n-1}\right\|+h^{k+1}+\tau^{2}\right)\left\|\xi_{p}^{n+1}\right\| .
$$

Using (5.8), the Lipschitz continuity of $\kappa(\cdot)^{-1}$ and Schwarz inequality again leads to

$$
T_{2} \leq C\left(\left\|\boldsymbol{\eta}_{\boldsymbol{u}}^{\boldsymbol{n}+\mathbf{1}}\right\|+\left\|\phi^{n+1}-\phi_{h}^{n+1}\right\|\right)\left\|\boldsymbol{\xi}_{\boldsymbol{u}}^{n+\mathbf{1}}\right\| \leq C\left(h^{k+1}+\left\|e_{\phi}^{n+1}\right\|\right)\left\|\boldsymbol{\xi}_{\boldsymbol{u}}^{\boldsymbol{n}+\mathbf{1}}\right\|,
$$

where the second step requires Lemma 5.3. As for $T_{3}$, it will be a little complicated. Using(4.13) and taking $\boldsymbol{\theta}=\boldsymbol{\xi}_{\boldsymbol{u}}^{\boldsymbol{n}-\boldsymbol{1}}$ in (5.39), we can obtain that

$$
\begin{aligned}
\frac{1}{4} \mathcal{L}^{d}\left(\boldsymbol{\xi}_{u}^{n-1}, \xi_{p}^{n+1}\right) & =-\frac{1}{4} \mathcal{D}\left(\xi_{p}^{n+1}, \boldsymbol{\xi}_{u}^{n-1}\right) \\
& =-\frac{1}{4}\left(\frac{\mu}{\kappa\left(\phi^{n+1}\right)} \mathbf{u}^{\mathbf{n}+\mathbf{1}}-\frac{\mu}{\kappa\left(\phi_{h}^{n+1}\right)} \mathbf{u}_{\mathbf{h}}^{\mathbf{n}+\mathbf{1}}, \boldsymbol{\xi}_{u}^{n-1}\right)-\frac{1}{4} \mathcal{D}\left(\eta_{p}^{n+1}, \boldsymbol{\xi}_{u}^{n-1}\right) .
\end{aligned}
$$

For the first term on the right-hand side, we have

$$
\begin{aligned}
& -\frac{1}{4}\left(\frac{\mu}{\kappa\left(\phi^{n+1}\right)} \mathbf{u}^{\mathbf{n}+\mathbf{1}}-\frac{\mu}{\kappa\left(\phi_{h}^{n+1}\right)} \mathbf{u}_{\mathbf{h}}^{\mathbf{n}+\mathbf{1}}, \boldsymbol{\xi}_{\boldsymbol{u}}^{n-\mathbf{1}}\right) \\
& \quad=-\frac{1}{4}\left(\left(\frac{\mu}{\kappa\left(\phi^{n+1}\right)}-\frac{\mu}{\kappa\left(\phi_{h}^{n+1}\right)}\right) \mathbf{u}_{\mathbf{h}}^{\mathbf{n}+\mathbf{1}}, \boldsymbol{\xi}_{\boldsymbol{u}}^{\mathbf{n - 1}}\right)-\frac{1}{4}\left(\frac{\mu}{\kappa\left(\phi^{n+1}\right)}\left(\mathbf{u}^{\mathbf{n}+\mathbf{1}}-\mathbf{u}_{\mathbf{h}}^{\mathbf{n}+\mathbf{1}}\right), \boldsymbol{\xi}_{\boldsymbol{u}}^{n-1}\right) .
\end{aligned}
$$

Then we obtain

$$
T_{3} \leq C\left\|e_{\phi}^{n+1}\right\|\left\|\xi_{u}^{n-1}\right\|+\frac{1}{4}\left\|\sqrt{\left(\frac{\mu}{\kappa\left(\phi^{n+1}\right)}\right)} \xi_{u}^{n+1}\right\|\left\|\sqrt{\left(\frac{\mu}{\kappa\left(\phi^{n-1}\right)}\right)} \xi_{u}^{n-1}\right\|
$$




$$
+\frac{1}{4}\left\|\sqrt{\left(\frac{\mu}{\kappa\left(\phi^{n+1}\right)}\right)} \eta_{u}^{n+1}\right\|\left\|\sqrt{\left(\frac{\mu}{\kappa\left(\phi^{n-1}\right)}\right)} \boldsymbol{\xi}_{u}^{n-1}\right\|+C h^{k+1}\left\|p^{n+1}\right\|_{k+2}\left\|\boldsymbol{\xi}_{u}^{n-1}\right\|,
$$

with the monotonicity of $\phi_{h}$. The estimate of $T_{4}$ follows from Lemma 3.3,

$$
T_{4} \leq C h^{k+1}\left\|p^{n+1}\right\|_{k+2}\left\|\boldsymbol{\xi}_{u}^{n+1}\right\| .
$$

Obviously,

$$
T_{5} \leq C \tau^{2}\left\|\xi_{p}^{n+1}\right\|
$$

Substituting the above estimates into (5.48), we obtain

$$
\begin{aligned}
\frac{\gamma}{2} \| \xi_{p}^{n+1} & \left\|^{2}+\frac{\gamma}{2}\right\| \xi_{p}^{n+1}-\xi_{p}^{n}\left\|^{2}-\frac{\gamma}{2}\right\| \xi_{p}^{n}\left\|^{2}+\frac{3 \tau}{4}\right\| \sqrt{\frac{\mu}{\kappa\left(\phi^{n+1}\right)}} \boldsymbol{\xi}_{\boldsymbol{u}}^{n+\mathbf{1}} \|^{2} \\
\leq & C \tau\left(\left\|e_{\phi}^{n+1}\right\|+\left\|e_{\phi}^{n}\right\|+\left\|\xi_{c}^{n}\right\|+\left\|\xi_{c}^{n-1}\right\|+h^{k+1}+\tau^{2}\right)\left\|\xi_{p}^{n+1}\right\| \\
& +C \tau\left(h^{k+1}+\left\|e_{\phi}^{n+1}\right\|\right)\left\|\boldsymbol{\xi}_{\boldsymbol{u}}^{n+\mathbf{1}}\right\|+C \tau\left(h^{k+1}+\left\|e_{\phi}^{n+1}\right\|\right)\left\|\boldsymbol{\xi}_{\boldsymbol{u}}^{n-1}\right\| \\
& +\frac{\tau}{4}\left\|\sqrt{\frac{\mu}{\kappa\left(\phi^{n+1}\right)}} \boldsymbol{\xi}_{\boldsymbol{u}}^{n+\mathbf{1}}\right\|\left\|\sqrt{\frac{\mu}{\kappa\left(\phi^{n-1}\right)}} \boldsymbol{\xi}_{\boldsymbol{u}}^{n-1}\right\|+\frac{\tau}{4}\left\|\sqrt{\frac{\mu}{\kappa\left(\phi^{n+1}\right)}} \boldsymbol{\eta}_{\boldsymbol{u}}^{n+1}\right\|\left\|\sqrt{\frac{\mu}{\kappa\left(\phi^{n-1}\right)}} \boldsymbol{\xi}_{\boldsymbol{u}}^{\boldsymbol{n}-\mathbf{1}}\right\| \\
\leq & C \tau\left(\left\|e_{\phi}^{n+1}-e_{\phi}^{n}\right\|^{2}+\left\|e_{\phi}^{n}\right\|^{2}+\left\|\xi_{c}^{n}\right\|^{2}+\left\|\xi_{c}^{n-1}\right\|^{2}+\left\|\xi_{p}^{n+1}-\xi_{p}^{n}\right\|^{2}+\left\|\xi_{p}^{n}\right\|^{2}+h^{2 k+2}+\tau^{4}\right) \\
& +\frac{\tau}{4}\left\|\sqrt{\frac{\mu}{\kappa\left(\phi^{n+1}\right)}} \boldsymbol{\xi}_{u}^{n+1}\right\|^{2}+\frac{\tau}{4}\left\|\sqrt{\frac{\mu}{\kappa\left(\phi^{n-1}\right)}} \boldsymbol{\xi}_{u}^{n-1}\right\|^{2} .
\end{aligned}
$$

Taking $n=-1$ and $\boldsymbol{\theta}=\boldsymbol{\xi}_{\boldsymbol{u}}^{\mathbf{0}}$ in (5.39), we have

$$
\left(\frac{\mu}{\kappa\left(\phi_{0}\right)} \boldsymbol{\xi}_{u}^{0}, \boldsymbol{\xi}_{u}^{\mathbf{0}}\right)=\left(\frac{\mu}{\kappa\left(\phi_{0}\right)} \boldsymbol{\eta}_{u}^{\mathbf{0}}, \boldsymbol{\xi}_{\boldsymbol{u}}^{\mathbf{0}}\right)-D\left(\eta_{p}^{0}, \boldsymbol{\xi}_{u}^{0}\right)
$$

By Schwarz inequality, Lemmas 3.3 and 5.3, we obtain

$$
\left\|\sqrt{\frac{\mu}{\kappa\left(\phi_{0}\right)}} \boldsymbol{\xi}_{\boldsymbol{u}}^{\mathbf{0}}\right\|^{2} \leq C h^{k+1}\left\|\boldsymbol{\xi}_{\boldsymbol{u}}^{\mathbf{0}}\right\|+C h^{k+1}\left\|p_{0}\right\|_{k+2}\left\|\boldsymbol{\xi}_{\boldsymbol{u}}^{\mathbf{0}}\right\| \leq C h^{2 k+2}+\varepsilon\left\|\sqrt{\frac{\mu}{\kappa\left(\phi_{0}\right)}} \boldsymbol{\xi}_{\boldsymbol{u}}^{\mathbf{0}}\right\|^{2},
$$

leading to

$$
\left\|\boldsymbol{\xi}_{u}^{0}\right\|^{2} \leq C h^{2 k+2}
$$

Summing (5.49) over $n$, we can get

$$
\begin{gathered}
\frac{\gamma}{2}\left\|\xi_{p}^{n+1}\right\|^{2}+\frac{\gamma}{2} \sum_{m=1}^{n}\left\|\xi_{p}^{m+1}-\xi_{p}^{m}\right\|^{2}+\frac{\tau}{4} \sum_{m=1}^{n}\left\|\sqrt{\frac{\mu}{\kappa\left(\phi^{m+1}\right)}} \boldsymbol{\xi}_{\boldsymbol{u}}^{m+1}\right\|^{2} \\
\leq C \tau \sum_{m=1}^{n}\left(\left\|e_{\phi}^{m+1}-e_{\phi}^{m}\right\|^{2}+\left\|e_{\phi}^{m}\right\|^{2}+\left\|\xi_{c}^{m}\right\|^{2}+\left\|\xi_{c}^{m-1}\right\|^{2}+\left\|\xi_{p}^{m+1}-\xi_{p}^{m}\right\|^{2}+\left\|\xi_{p}^{m}\right\|^{2}\right) \\
+C\left(h^{2 k+2}+\tau^{4}\right)+\frac{\tau}{4}\left\|\sqrt{\frac{\mu}{\kappa\left(\phi^{1}\right)}} \boldsymbol{\xi}_{\boldsymbol{u}}^{1}\right\|^{2}+\frac{\gamma}{2}\left\|\xi_{p}^{1}\right\|^{2} .
\end{gathered}
$$

Step 3. Taking $v=\xi_{c}^{n+1}$ in (5.40) and $\mathbf{w}=\frac{3}{4} \boldsymbol{\xi}_{\boldsymbol{s}}^{\boldsymbol{n}+\mathbf{1}}$ in (5.41), then summing these two equations, we have

$$
\left(\frac{\left(\xi_{c}^{n+1}-\xi_{c}^{n}\right) \phi_{h}^{n+1}}{\tau}, \xi_{c}^{n+1}\right)+\frac{3}{4}\left(\left(\phi_{h}^{n+1} \mathbf{D}\left(\mathbf{u}_{\mathbf{h}}^{\mathbf{n}+\mathbf{1}, \mathbf{M}}\right)\right)^{-1} \boldsymbol{\xi}_{s}^{n+\mathbf{1}}, \boldsymbol{\xi}_{s}^{n+\mathbf{1}}\right)=\sum_{i=1}^{7} R_{i},
$$


where

$$
\begin{aligned}
R_{1}= & \left(\frac{\left(\eta_{c}^{n+1}-\eta_{c}^{n}\right) \phi_{h}^{n+1}}{\tau}, \xi_{c}^{n+1}\right)-\left(\frac{\left(c_{f}^{n+1}-c_{f}^{n}\right) e_{\phi}^{n+1}}{\tau}, \xi_{c}^{n+1}\right)-\left(\frac{\left(e_{\phi}^{n+1}-e_{\phi}^{n}\right) c_{f}^{n}}{\tau}, \xi_{c}^{n+1}\right) \\
& -\left(\frac{\left(\phi_{h}^{n+1}-\phi_{h}^{n}\right) \xi_{c}^{n}}{\tau}, \xi_{c}^{n+1}\right)+\left(\frac{\left(\phi_{h}^{n+1}-\phi_{h}^{n}\right) \eta_{c}^{n}}{\tau}, \xi_{c}^{n+1}\right), \\
R_{2}= & \frac{3}{4}\left(\left(\left(\phi_{h}^{n+1} \mathbf{D}\left(\mathbf{u}_{\mathbf{h}}^{\mathbf{n}+\mathbf{1}, \mathbf{M}}\right)\right)^{-1}-\left(\phi^{n+1} \mathbf{D}\left(\mathbf{u}^{\mathbf{n}+\mathbf{1}}\right)\right)^{-1}\right) \mathbf{s}^{\mathbf{n}+\mathbf{1}}, \boldsymbol{\xi}_{s}^{n+\mathbf{1}}\right) \\
& +\frac{3}{4}\left(\left(\left(\phi^{n+1}\right)^{-1} \mathbf{M}_{\mathbf{e}}\left(\mathbf{u}^{\mathbf{n}+\mathbf{1}}\right)-\left(\phi_{h}^{n+1}\right)^{-1} \mathbf{M}_{\mathbf{e}}\left(\mathbf{u}_{\mathbf{h}}^{\mathbf{n}+\mathbf{1}}\right)\right) c_{f}^{n+1}, \boldsymbol{\xi}_{s}^{n+\mathbf{1}}\right), \\
R_{3}= & \frac{1}{4} \mathcal{L}^{d}\left(\boldsymbol{\xi}_{s}^{n-1}, \xi_{c}^{n+1}\right), \\
R_{4}= & -\frac{3}{4} \mathcal{D}\left(\eta_{c}^{n+1}, \boldsymbol{\xi}_{s}^{n+\mathbf{1}}\right), \\
R_{5}= & \frac{3}{4}\left(\left(\phi_{h}^{n+1} \mathbf{D}\left(\mathbf{u}_{\mathbf{h}}^{\mathbf{n}+\mathbf{1}, \mathbf{M}}\right)\right)^{-1} \boldsymbol{\eta}_{s}^{n+1}, \boldsymbol{\xi}_{s}^{n+\mathbf{1}}\right)+\frac{3}{4}\left(\left(\phi_{h}^{n+1}\right)^{-1} \mathbf{M}_{\mathbf{e}}\left(\mathbf{u}_{\mathbf{h}}^{\mathbf{n}+\mathbf{1}}\right) e_{c}^{n+1}, \boldsymbol{\xi}_{s}^{n+\mathbf{1}}\right), \\
R_{6}= & \frac{3}{2}\left(f_{p}^{n} e_{c}^{n}, \xi_{c}^{n+1}\right)-\frac{1}{2}\left(f_{p}^{n-1} e_{c}^{n-1}, \xi_{c}^{n+1}\right)+\left(\varsigma_{2}^{n}, \xi_{c}^{n+1}\right), \\
R_{7}= & -\frac{3}{2} A\left(a_{v}\left(\phi^{n}\right) c_{f}^{n}-a_{v}\left(\phi_{h}^{n}\right) c_{h}^{n}, \xi_{c}^{n+1}\right)+\frac{1}{2} A\left(a_{v}\left(\phi^{n-1}\right) c_{f}^{n-1}-a_{v}\left(\phi_{h}^{n-1}\right) c_{h}^{n-1}, \xi_{c}^{n+1}\right) .
\end{aligned}
$$

Now we estimate $R_{i}(i=1,2,3,4,5,6,7)$ term by term. The estimate is similar to the situation with $r=1$ except $R_{3}$.

$$
\begin{aligned}
& R_{1} \leq C\left(h^{k+1}+\left\|e_{\phi}^{n+1}\right\|+\left\|e_{\phi}^{n}\right\|+\left\|\xi_{c}^{n}\right\|+\left\|\xi_{c}^{n-1}\right\|+\tau^{2}\right)\left\|\xi_{c}^{n+1}\right\|, \\
& R_{2} \leq C\left(\left\|\boldsymbol{\xi}_{u}^{n+1}\right\|+\left\|e_{\phi}^{n+1}\right\|+h^{k+1}\right)\left\|\boldsymbol{\xi}_{s}^{n+1}\right\| \\
& R_{4} \leq C h^{k+1}\left\|c_{f}^{n+1}\right\|_{k+2}\left\|\boldsymbol{\xi}_{s}^{n+\mathbf{1}}\right\| \\
& R_{5} \leq C\left(\left\|\xi_{c}^{n+1}\right\|+h^{k+1}\right)\left\|\boldsymbol{\xi}_{s}^{n+1}\right\| \\
& R_{6} \leq C\left(\left\|\xi_{c}^{n-1}\right\|+\left\|\xi_{c}^{n}\right\|+h^{k+1}+\tau^{2}\right)\left\|\xi_{c}^{n+1}\right\| \\
& R_{7} \leq C\left(\left\|\xi_{c}^{n}\right\|+\left\|\xi_{c}^{n-1}\right\|+\left\|e_{\phi}^{n}\right\|+\left\|e_{\phi}^{n-1}\right\|+h^{k+1}\right)\left\|\xi_{c}^{n+1}\right\| .
\end{aligned}
$$

Next, we estimate $R_{3}$. Applying (4.13) and taking $\mathbf{w}=\boldsymbol{\xi}_{\boldsymbol{s}}^{\boldsymbol{n}-\mathbf{1}}$ in (5.41) to get

$$
\begin{aligned}
R_{3}= & -\frac{1}{4} \mathcal{D}\left(\xi_{c}^{n+1}, \boldsymbol{\xi}_{s}^{n-\mathbf{1}}\right) \\
= & -\frac{1}{4}\left(\left(\phi^{n+1} \mathbf{D}\left(\mathbf{u}^{\mathbf{n}+\mathbf{1}}\right)\right)^{-1} \mathbf{s}^{\mathbf{n}+\mathbf{1}}-\left(\phi_{h}^{n+1} \mathbf{D}\left(\mathbf{u}_{\mathbf{h}}^{\mathbf{n}+\mathbf{1}, \mathbf{M}}\right)\right)^{-1} \mathbf{s}_{\mathbf{h}}^{\mathbf{n}+\mathbf{1}}, \boldsymbol{\xi}_{s}^{n-\mathbf{1}}\right) \\
& +\frac{1}{4}\left(\left(\phi^{n+1}\right)^{-1} \mathbf{M}_{\mathbf{e}}\left(\mathbf{u}^{\mathbf{n}+\mathbf{1}}\right) c_{f}^{n+1}-\left(\phi_{h}^{n+1}\right)^{-1} \mathbf{M}_{\mathbf{e}}\left(\mathbf{u}_{\mathbf{h}}^{\mathbf{n}+\mathbf{1}}\right) c_{h}^{n+1}, \boldsymbol{\xi}_{s}^{n-\mathbf{1}}\right)-\frac{1}{4} \mathcal{D}\left(\eta_{c}^{n+1}, \boldsymbol{\xi}_{s}^{n-\mathbf{1}}\right) .
\end{aligned}
$$

In particular,

$$
\begin{aligned}
-\frac{1}{4} & \left(\left(\phi^{n+1} \mathbf{D}\left(\mathbf{u}^{\mathbf{n}+\mathbf{1}}\right)\right)^{-1} \mathbf{s}^{\mathbf{n}+\mathbf{1}}-\left(\phi_{h}^{n+1} \mathbf{D}\left(\mathbf{u}_{\mathbf{h}}^{\mathbf{n}+\mathbf{1}, \mathbf{M}}\right)\right)^{-1} \mathbf{s}_{\mathbf{h}}^{\mathbf{n}+\mathbf{1}}, \boldsymbol{\xi}_{s}^{n-\mathbf{1}}\right) \\
& =-\frac{1}{4}\left(\left(\phi_{h}^{n+1} \mathbf{D}\left(\mathbf{u}_{\mathbf{h}}^{\mathbf{n}+\mathbf{1}, \mathbf{M}}\right)\right)^{-1} \boldsymbol{\xi}_{s}^{n+\mathbf{1}}, \boldsymbol{\xi}_{s}^{n-\mathbf{1}}\right)+\frac{1}{4}\left(\left(\phi_{h}^{n+1} \mathbf{D}\left(\mathbf{u}_{\mathbf{h}}^{\mathbf{n}+\mathbf{1}, \mathbf{M}}\right)\right)^{-1} \boldsymbol{\eta}_{s}^{n+\mathbf{1}}, \boldsymbol{\xi}_{s}^{n-\mathbf{1}}\right)
\end{aligned}
$$




$$
\begin{aligned}
& -\frac{1}{4}\left(\left(\left(\phi^{n+1} \mathbf{D}\left(\mathbf{u}^{\mathbf{n}+\mathbf{1}}\right)\right)^{-1}-\left(\phi_{h}^{n+1} \mathbf{D}\left(\mathbf{u}_{\mathbf{h}}^{\mathbf{n}+\mathbf{1}, \mathbf{M}}\right)\right)^{-1}\right) \mathbf{s}^{\mathbf{n + 1}}, \boldsymbol{\xi}_{\boldsymbol{s}}^{\mathbf{n - 1}}\right) \\
:= & \Lambda_{1}+\Lambda_{2}+\Lambda_{3} .
\end{aligned}
$$

Here we define a new inner product $\langle\mathbf{v}, \mathbf{v}\rangle=\left((\phi \mathbf{D}(\mathbf{u}))^{-\mathbf{1}} \mathbf{v}, \mathbf{v}\right)$, and applying Schwarz inequality, the monotonicity of $\phi_{h}$ and Young's inequality to $\Lambda_{1}$, we get

$$
\left|\Lambda_{1}\right| \leq \frac{1}{8}\left(\left(\phi_{h}^{n+1} \mathbf{D}\left(\mathbf{u}_{\mathbf{h}}^{\mathbf{n}+\mathbf{1}, \mathbf{M}}\right)\right)^{-1} \boldsymbol{\xi}_{\boldsymbol{s}}^{\mathbf{n + 1}}, \boldsymbol{\xi}_{\boldsymbol{s}}^{\mathbf{n + 1}}\right)+\frac{1}{8}\left(\left(\phi_{h}^{n-1} \mathbf{D}\left(\mathbf{u}_{\mathbf{h}}^{\mathbf{n}+\mathbf{1}, \mathbf{M}}\right)\right)^{-1} \boldsymbol{\xi}_{\boldsymbol{s}}^{\boldsymbol{n}-\mathbf{1}}, \boldsymbol{\xi}_{\boldsymbol{s}}^{\boldsymbol{n}-\mathbf{1}}\right)=\Theta+\widetilde{\Theta}
$$

where

$$
\begin{aligned}
& \Theta=\frac{1}{8}\left(\left\|\left(\phi_{h}^{n+1} \mathbf{D}\left(\mathbf{u}_{\mathbf{h}}^{\mathbf{n}+\mathbf{1}, \mathbf{M}}\right)\right)^{-1 / 2} \boldsymbol{\xi}_{\boldsymbol{s}}^{\mathbf{n + 1}}\right\|^{2}+\left\|\left(\phi_{h}^{n-1} \mathbf{D}\left(\mathbf{u}_{\mathbf{h}}^{\mathbf{n}-\mathbf{1}, \mathbf{M}}\right)\right)^{-1 / 2} \boldsymbol{\xi}_{\boldsymbol{s}}^{\mathbf{n}-\mathbf{1}}\right\|^{2}\right), \\
& \widetilde{\Theta}=\frac{1}{8}\left(\left(\left(\phi_{h}^{n-1} \mathbf{D}\left(\mathbf{u}_{\mathbf{h}}^{\mathbf{n}+\mathbf{1}, \mathbf{M}}\right)\right)^{-1}-\left(\phi_{h}^{n-1} \mathbf{D}\left(\mathbf{u}_{\mathbf{h}}^{\mathbf{n}-\mathbf{1}, \mathbf{M}}\right)\right)^{-1}\right) \boldsymbol{\xi}_{\boldsymbol{s}}^{\boldsymbol{n}-\mathbf{1}}, \boldsymbol{\xi}_{\boldsymbol{s}}^{\mathbf{n}-\mathbf{1}}\right) .
\end{aligned}
$$

Notice that

$$
\begin{aligned}
\left(\phi_{h}^{n-1} \mathbf{D}\left(\mathbf{u}_{\mathbf{h}}^{\mathbf{n}+\mathbf{1}, \mathbf{M}}\right)\right)^{-1}-\left(\phi_{h}^{n-1} \mathbf{D}\left(\mathbf{u}_{\mathbf{h}}^{\mathbf{n}-\mathbf{1}, \mathbf{M}}\right)\right)^{-1} & \\
= & {\left[\left(\phi_{h}^{n-1} \mathbf{D}\left(\mathbf{u}^{\mathbf{n}+\mathbf{1}}\right)\right)^{-1}-\left(\phi_{h}^{n-1} \mathbf{D}\left(\mathbf{u}^{\mathbf{n}-\mathbf{1}}\right)\right)^{-1}\right]-\left[\left(\phi_{h}^{n-1} \mathbf{D}\left(\mathbf{u}^{\mathbf{n}+\mathbf{1}}\right)\right)^{-1}-\left(\phi_{h}^{n-1} \mathbf{D}\left(\mathbf{u}_{\mathbf{h}}^{\mathbf{n}+\mathbf{1}, \mathbf{M}}\right)\right)^{-1}\right] } \\
& +\left[\left(\phi_{h}^{n-1} \mathbf{D}\left(\mathbf{u}^{\mathbf{n}-\mathbf{1}}\right)\right)^{-1}-\left(\phi_{h}^{n-1} \mathbf{D}\left(\mathbf{u}_{\mathbf{h}}^{\mathbf{n}-\mathbf{1}, \mathbf{M}}\right)\right)^{-1}\right]
\end{aligned}
$$

By the a priori assumption, we can further get

$$
\begin{aligned}
|\widetilde{\Theta}| & \leq C\left(\tau+\left\|\mathbf{e}_{\mathbf{u}}^{\mathbf{n}+\mathbf{1}}\right\|_{\infty}+\left\|\mathbf{e}_{\mathbf{u}}^{\mathbf{n}-\mathbf{1}}\right\|_{\infty}\right)\left\|\boldsymbol{\xi}_{\boldsymbol{s}}^{\boldsymbol{n}-\mathbf{1}}\right\|^{2} \leq C\left(\tau+h^{\delta}\right)\left\|\boldsymbol{\xi}_{\boldsymbol{s}}^{\boldsymbol{n}-\mathbf{1}}\right\|^{2} \\
& \leq \frac{1}{8}\left\|\left(\phi_{h}^{n-1} \mathbf{D}\left(\mathbf{u}_{\mathbf{h}}^{\mathbf{n}-\mathbf{1}, \mathbf{M}}\right)\right)^{-1 / 2} \boldsymbol{\xi}_{\boldsymbol{s}}^{\boldsymbol{n}-\mathbf{1}}\right\|^{2}
\end{aligned}
$$

Then we have

$$
R_{3} \leq \Theta+\frac{1}{8}\left\|\left(\phi_{h}^{n-1} \mathbf{D}\left(\mathbf{u}_{\mathbf{h}}^{\mathbf{n}-\mathbf{1}, \mathbf{M}}\right)\right)^{-1 / 2} \boldsymbol{\xi}_{\boldsymbol{s}}^{\boldsymbol{n}-\mathbf{1}}\right\|^{2}+C\left(\left\|\xi_{c}^{n+1}\right\|+\left\|e_{\phi}^{n+1}\right\|+\left\|\boldsymbol{\xi}_{\boldsymbol{u}}^{\boldsymbol{n}+\mathbf{1}}\right\|+h^{k+1}\right)\left\|\boldsymbol{\xi}_{\boldsymbol{s}}^{\boldsymbol{n}-\mathbf{1}}\right\|
$$

by using the boundedness of $\phi_{h}^{n+1}, \mathbf{D}\left(\mathbf{u}_{\mathbf{h}}^{\mathbf{n}+\mathbf{1}, \mathbf{M}}\right)$ and $\mathbf{M}_{\mathbf{e}}\left(\mathbf{u}_{\mathbf{h}}^{\mathbf{n}+\mathbf{1}}\right)$ and Lemma 3.3. Substituting the above estimates into (5.52), we have

$$
\begin{aligned}
\frac{1}{2} \| & \sqrt{\phi_{h}^{n+1}} \xi_{c}^{n+1}\left\|^{2}+\frac{1}{2}\right\| \sqrt{\phi_{h}^{n+1}}\left(\xi_{c}^{n+1}-\xi_{c}^{n}\right)\left\|^{2}-\frac{1}{2}\right\| \sqrt{\phi_{h}^{n}} \xi_{c}^{n} \|^{2} \\
& +\frac{3}{4} \tau\left\|\left(\phi_{h}^{n+1} \mathbf{D}\left(\mathbf{u}_{\mathbf{h}}^{\mathbf{n}+\mathbf{1}, \mathbf{M}}\right)\right)^{-1 / 2} \boldsymbol{\xi}_{s}^{\mathbf{n + 1}}\right\|^{2} \\
\leq & C \tau\left(\left\|e_{\phi}^{n+1}-e_{\phi}^{n}\right\|^{2}+\left\|e_{\phi}^{n}\right\|^{2}+\left\|e_{\phi}^{n-1}\right\|^{2}+\left\|\xi_{c}^{n+1}-\xi_{c}^{n}\right\|^{2}+\left\|\xi_{c}^{n}\right\|^{2}+\left\|\xi_{c}^{n-1}\right\|^{2}+\left\|\boldsymbol{\xi}_{\boldsymbol{u}}^{\mathbf{n}+\mathbf{1}}\right\|^{2}\right) \\
& +\varepsilon \tau\left\|\left(\phi_{h}^{n+1} \mathbf{D}\left(\mathbf{u}_{\mathbf{h}}^{\mathbf{n}+\mathbf{1}, \mathbf{M}}\right)\right)^{-1 / 2} \boldsymbol{\xi}_{\boldsymbol{s}}^{\mathbf{n}+\mathbf{1}}\right\|^{2}+\varepsilon \tau\left\|\left(\phi_{h}^{n-1} \mathbf{D}\left(\mathbf{u}_{\mathbf{h}}^{\mathbf{n}-\mathbf{1}, \mathbf{M}}\right)\right)^{-1 / 2} \boldsymbol{\xi}_{\boldsymbol{s}}^{\mathbf{n}-\mathbf{1}}\right\|^{2} \|^{2}
\end{aligned}
$$




$$
+\frac{1}{8} \tau\left\|\left(\phi_{h}^{n-1} \mathbf{D}\left(\mathbf{u}_{\mathbf{h}}^{\mathbf{n}-1, \mathbf{M}}\right)\right)^{-1 / 2} \boldsymbol{\xi}_{s}^{n-1}\right\|^{2}+\tau \Theta+C \tau\left(h^{2 k+2}+\tau^{4}\right)
$$

where the treatment of the third term on the left-hand side is similar to (4.43). Taking $n=-1$ and $\mathbf{w}=\boldsymbol{\xi}_{\boldsymbol{s}}^{\mathbf{0}}$ in (5.41) to get

$$
\begin{aligned}
\left(\left(\phi_{0} \mathbf{D}\left(\mathbf{u}_{\mathbf{h}}^{\mathbf{0}, \mathbf{M}}\right)\right)^{-1} \boldsymbol{\xi}_{s}^{\mathbf{0}}, \boldsymbol{\xi}_{s}^{\mathbf{0}}\right)= & \left(\left(\phi_{0} \mathbf{D}\left(\mathbf{u}_{\mathbf{h}}^{\mathbf{0}, \mathbf{M}}\right)\right)^{-1} \boldsymbol{\eta}_{s}^{\mathbf{0}}, \boldsymbol{\xi}_{s}^{\mathbf{0}}\right)-\left(\left(\left(\phi_{0} \mathbf{D}\left(\mathbf{u}^{\mathbf{0}}\right)\right)^{-1}-\left(\phi_{0} \mathbf{D}\left(\mathbf{u}_{\mathbf{h}}^{\mathbf{0}, \mathbf{M}}\right)\right)^{-1}\right) \mathbf{s}_{\mathbf{0}}, \boldsymbol{\xi}_{s}^{\mathbf{0}}\right) \\
& +\left(\left(\phi_{0}\right)^{-1} \mathbf{M}_{\mathbf{e}}\left(\mathbf{u}^{\mathbf{0}}\right) c_{f}^{0}-\left(\phi_{0}\right)^{-1} \mathbf{M}_{\mathbf{e}}\left(\mathbf{u}_{\mathbf{h}}^{\mathbf{0}}\right) c_{h}^{0}, \boldsymbol{\xi}_{s}^{\mathbf{0}}\right)-\mathcal{D}\left(\eta_{c}^{0}, \boldsymbol{\xi}_{s}^{\mathbf{0}}\right) .
\end{aligned}
$$

Then we have

$$
\begin{aligned}
\left\|\left(\phi_{0} \mathbf{D}\left(\mathbf{u}_{\mathbf{h}}^{\mathbf{0}, \mathbf{M}}\right)\right)^{-1 / 2} \boldsymbol{\xi}_{\boldsymbol{s}}^{\mathbf{0}}\right\|^{2} \leq & C\left\|\boldsymbol{\eta}_{\boldsymbol{s}}^{\mathbf{0}}\right\|\left\|\boldsymbol{\xi}_{\boldsymbol{s}}^{\mathbf{0}}\right\|+C\left\|\mathbf{e}_{\mathbf{u}}^{\mathbf{0}}\right\|\left\|\boldsymbol{\xi}_{\boldsymbol{s}}^{\mathbf{0}}\right\|+C\left\|e_{c}^{0}\right\|\left\|\boldsymbol{\xi}_{\boldsymbol{s}}^{\mathbf{0}}\right\|+C h^{k+1}\left\|c_{f}^{0}\right\|_{k+2}\left\|\boldsymbol{\xi}_{\boldsymbol{s}}^{\mathbf{0}}\right\| \\
\leq & C\left\|\boldsymbol{\eta}_{\boldsymbol{s}}^{\mathbf{0}}\right\|\left\|\boldsymbol{\xi}_{\boldsymbol{s}}^{\mathbf{0}}\right\|+C\left\|\boldsymbol{\xi}_{\boldsymbol{u}}^{\mathbf{0}}\right\|\left\|\boldsymbol{\xi}_{\boldsymbol{s}}^{\mathbf{0}}\right\|+C\left\|\boldsymbol{\eta}_{\boldsymbol{u}}^{\mathbf{0}}\right\|\left\|\boldsymbol{\xi}_{\boldsymbol{s}}^{\mathbf{0}}\right\|+C\left\|\eta_{c}^{0}\right\|\left\|\boldsymbol{\xi}_{\boldsymbol{s}}^{\mathbf{0}}\right\| \\
& +C h^{k+1}\left\|c_{f}^{0}\right\|_{k+2}\left\|\boldsymbol{\xi}_{\boldsymbol{s}}^{\mathbf{0}}\right\| \\
\leq & C h^{2 k+2}+\varepsilon\left\|\left(\phi_{0} \mathbf{D}\left(\mathbf{u}_{\mathbf{h}}^{\mathbf{0}, \mathbf{M}}\right)\right)^{-1 / 2} \boldsymbol{\xi}_{\boldsymbol{s}}^{\mathbf{0}}\right\|^{2}
\end{aligned}
$$

where in the first step we use Lemma 3.3 and the Lipschitz continuity of $\mathbf{D}(\cdot)^{-\mathbf{1}}$ and $\mathbf{M}_{\mathbf{e}}(\cdot)$ given in (4.28) and (4.30) while the third step is based on Lemma 5.3 and the estimate of $\boldsymbol{\xi}_{\boldsymbol{u}}^{\mathbf{0}}$ in (5.50). It is easy to obtain

$$
\left\|\boldsymbol{\xi}_{\boldsymbol{s}}^{\mathbf{0}}\right\|^{2} \leq C h^{2 k+2}
$$

Taking $\varepsilon=\frac{1}{8}$, summing (5.55) over $n$ and using (5.51) to obtain

$$
\begin{aligned}
& \frac{1}{2}\left\|\sqrt{\phi_{h}^{n+1}} \xi_{c}^{n+1}\right\|^{2}+\frac{1}{8} \tau \sum_{m=1}^{n}\left\|\left(\phi_{h}^{m+1} \mathbf{D}\left(\mathbf{u}_{\mathbf{h}}^{\mathbf{m}+\mathbf{1}, \mathbf{M}}\right)\right)^{-1 / 2} \boldsymbol{\xi}_{s}^{m+\mathbf{1}}\right\|^{2} \\
& \leq C \tau \sum_{m=1}^{n}\left(\left\|e_{\phi}^{m+1}-e_{\phi}^{m}\right\|^{2}+\left\|e_{\phi}^{m}\right\|^{2}+\left\|e_{\phi}^{m-1}\right\|^{2}\right) \\
&+C \tau \sum_{m=1}^{n}\left(\left\|\xi_{c}^{m}\right\|^{2}+\left\|\xi_{c}^{m-1}\right\|^{2}+\left\|\xi_{p}^{m+1}-\xi_{p}^{m}\right\|^{2}+\left\|\xi_{p}^{m}\right\|^{2}\right) \\
&+C\left(h^{2 k+2}+\tau^{4}\right)+\frac{3 \tau}{8}\left\|\left(\phi_{h}^{1} \mathbf{D}\left(\mathbf{u}_{\mathbf{h}}^{\mathbf{1}, \mathbf{M}}\right)\right)^{-1 / 2} \boldsymbol{\xi}_{\boldsymbol{s}}^{\mathbf{1}}\right\|^{2} \\
&+\frac{1}{2}\left\|\sqrt{\phi_{h}^{1}} \xi_{c}^{1}\right\|^{2}+C\left(\left\|\xi_{p}^{1}\right\|^{2}+\tau\left\|\left(\frac{\mu}{\kappa\left(\phi^{1}\right)}\right)^{1 / 2} \boldsymbol{\xi}_{u}^{\mathbf{1}}\right\|^{2}\right)
\end{aligned}
$$

Combining (5.46), (5.51) and (5.57), and under the condition $h^{2 k-3 \delta} \leq \tau \leq h^{\frac{2+3 \delta}{3}}$, we obtain

$$
\begin{aligned}
& \frac{1}{2}\left\|e_{\phi}^{n+1}\right\|^{2}+\frac{\gamma}{2}\left\|\xi_{p}^{n+1}\right\|^{2}+\frac{1}{2}\left\|\sqrt{\phi_{h}^{n+1}} \xi_{c}^{n+1}\right\|^{2}+\frac{1}{4} \tau \sum_{m=1}^{n}\left\|\left(\frac{\mu}{\kappa\left(\phi^{m+1}\right)}\right)^{1 / 2} \boldsymbol{\xi}_{u}^{m+\mathbf{1}}\right\|^{2} \\
& \quad+\frac{1}{8} \tau \sum_{m=1}^{n}\left\|\left(\phi_{h}^{m+1} \mathbf{D}\left(\mathbf{u}_{\mathbf{h}}^{\mathbf{m}+\mathbf{1}, \mathbf{M}}\right)\right)^{-1 / 2} \boldsymbol{\xi}_{s}^{m+\mathbf{1}}\right\|^{2}
\end{aligned}
$$




$$
\leq C \tau \sum_{m=1}^{n}\left(\left\|e_{\phi}^{m}\right\|^{2}+\left\|e_{\phi}^{m-1}\right\|^{2}+\left\|\xi_{c}^{m}\right\|^{2}+\left\|\xi_{c}^{m-1}\right\|^{2}+\left\|\xi_{p}^{m}\right\|^{2}\right)+C\left(h^{2 k+2}+\tau^{4}\right)+Q_{1},
$$

where

$$
\begin{aligned}
Q_{1}= & \frac{1}{2}\left\|e_{\phi}^{1}\right\|^{2}+\frac{1}{2}\left\|\sqrt{\phi_{h}^{1}} \xi_{c}^{1}\right\|^{2}+\frac{3 \tau}{8}\left\|\left(\phi_{h}^{1} \mathbf{D}\left(\mathbf{u}_{\mathbf{h}}^{\mathbf{1}, \mathbf{M}}\right)\right)^{-1 / 2} \boldsymbol{\xi}_{\boldsymbol{s}}^{\mathbf{1}}\right\|^{2} \\
& +C\left(\left\|\xi_{p}^{1}\right\|^{2}+\tau\left\|\left(\frac{\mu}{\kappa\left(\phi^{1}\right)}\right)^{1 / 2} \boldsymbol{\xi}_{u}^{1}\right\|^{2}\right) \leq C\left(h^{2 k+2}+\tau^{4}\right) .
\end{aligned}
$$

We can get Lemma 5.4 by applying Lemma 3.5 to (5.58).

And next we will proof (5.59). Since the second-order scheme starts with the first-order scheme, combining (5.22), (5.26) and (5.29), and taking $n=0$ leads to

$$
\begin{aligned}
\frac{1}{2}\left\|e_{\phi}^{1}\right\|^{2} & +\frac{\gamma}{2}\left\|\xi_{p}^{1}\right\|^{2}+\frac{1}{2}\left\|\sqrt{\phi_{h}^{1}} \xi_{c}^{1}\right\|^{2}+\tau\left\|\left(\frac{\mu}{\kappa\left(\phi_{h}^{1}\right)}\right)^{1 / 2} \boldsymbol{\xi}_{u}^{\mathbf{1}}\right\|^{2}+\tau\left\|\left(\phi_{h}^{1} \mathbf{D}\left(\mathbf{u}_{\mathbf{h}}^{\mathbf{1}, \mathbf{M}}\right)\right)^{-1 / 2} \boldsymbol{\xi}_{\boldsymbol{s}}^{\mathbf{1}}\right\|^{2} \\
\leq & C \tau\left(\left\|e_{\phi}^{1}\right\|+h^{k+1}+\tau\right)\left\|e_{\phi}^{1}\right\|+C \tau\left(\left\|e_{\phi}^{1}\right\|+h^{k+1}\right)\left\|\boldsymbol{\xi}_{u}^{\mathbf{1}}\right\| \\
& \left.+C \tau\left(\left\|e_{\phi}^{1}\right\|+h^{k+1}+\tau\right)\left\|\xi_{c}^{1}\right\|+C \tau\left(\left\|e_{\phi}^{1}\right\|+h^{k+1}+\tau\right)\right)\left\|\xi_{p}^{1}\right\| \\
& +C \tau\left(\left\|e_{\phi}^{1}\right\|+\left\|\xi_{c}^{1}\right\|+\left\|\boldsymbol{\xi}_{u}^{1}\right\|+h^{k+1}\right)\left\|\boldsymbol{\xi}_{s}^{1}\right\| \\
\leq & C \tau\left(\left\|e_{\phi}^{1}\right\|^{2}+\left\|\xi_{c}^{1}\right\|^{2}+\left\|\xi_{p}^{1}\right\|^{2}\right)+\varepsilon \tau\left\|\boldsymbol{\xi}_{s}^{\mathbf{1}}\right\|^{2}+\varepsilon\left(\left\|e_{\phi}^{1}\right\|^{2}+\left\|\xi_{c}^{1}\right\|^{2}+\left\|\xi_{p}^{1}\right\|^{2}\right)+C \tau h^{2 k+2}+C \tau^{4}
\end{aligned}
$$

where we use (5.26) again and $\tau$ is small enough. Therefore we obtain (5.59).

To complete this proof, we need to show the reasonability of the a priori assumption. In fact, it can be verified easily. Since $\left\|\boldsymbol{\xi}_{\boldsymbol{u}}^{0}\right\| \leq C h^{k+1}$, it follows from $k \geq 1$ and the approximation property (5.5) that

$$
\left\|\mathbf{e}_{\mathbf{u}}^{\mathbf{0}}\right\| \leq\left\|\boldsymbol{\xi}_{\boldsymbol{u}}^{\mathbf{0}}\right\|+\left\|\boldsymbol{\eta}_{\boldsymbol{u}}^{\mathbf{0}}\right\| \leq C h^{k+1} \leq h^{1+\delta},
$$

if $h$ is small enough. Assume (5.7) holds for any given $n$, then (5.9) holds with bounding constant independent of $n$ and $\tau$, which implies

$$
\left\|\mathbf{e}_{\mathbf{u}}^{\mathbf{n}+\mathbf{1}}\right\| \leq C \frac{\left(h^{k+1}+\tau^{2}\right)}{\tau^{1 / 2}} \leq h^{1+\delta},
$$

if $h$ is small enough. The a priori assumption is reasonable hence.

\section{NUMERICAL EXPERIMENTS}

In this section, we perform several numerical examples to illustrate the accuracy and capability of the fullydiscrete schemes (4.14)-(4.25) for wormhole propagations. A similar wormhole propagation problem were studied in $[10,22]$, where the modelling equations (2.1)-(2.4) were solved by the spectral deferred correction time method. The equation for the porosity was solved by an explicit scheme, and no stability has been proved in [10,22]. In this paper, we deal with the time scheme for the porosity as (4.14) and (4.20), introduce a variable for both the convection and diffusion terms in the concentration equation, finally prove the stability of the fully-discrete schemes. 
TABLE 1. Accuracy test for the stable implicit time scheme (4.14)-(4.18) and (4.20)-(4.25) of the wormhole propagations with parameters (6.1) in Example 6.1. The exact smooth solutions are chosen as (6.2). The computational domain $\Omega=[0,1] \times[0,1]$ is composed by $N \times N$ elements, and the final time is $T=0.2$. The first and second fully-discrete time scheme are employed with the time step $\tau=0.2 h, h=\frac{1}{N}$. Piecewise linear tensor product polynomials are employed in the LDG scheme.

\begin{tabular}{llllllllll}
\hline \hline Time scheme & $N$ & $\left\|p-p_{h}\right\|$ & Order & $\left\|u-u_{h}\right\|$ & Order & $\left\|c_{f}-c_{f}\right\|$ & Order & $\left\|\phi-\phi_{h}\right\|$ & Order \\
\hline \multirow{3}{*}{ First order } & 16 & $9.07 \mathrm{E}-3$ & - & $1.03 \mathrm{E}-1$ & - & $1.74 \mathrm{E}-3$ & - & $1.25 \mathrm{E}-3$ & - \\
& 32 & $3.13 \mathrm{E}-3$ & 1.53 & $3.84 \mathrm{E}-2$ & 1.42 & $6.91 \mathrm{E}-4$ & 1.33 & $6.26 \mathrm{E}-4$ & 1.00 \\
& 64 & $1.33 \mathrm{E}-3$ & 1.23 & $1.72 \mathrm{E}-2$ & 1.16 & $3.52 \mathrm{E}-4$ & 0.97 & $3.13 \mathrm{E}-4$ & 1.00 \\
& 128 & $6.31 \mathrm{E}-4$ & 1.07 & $8.35 \mathrm{E}-3$ & 1.04 & $1.78 \mathrm{E}-4$ & 0.98 & $1.56 \mathrm{E}-4$ & 1.00 \\
\hline \multirow{3}{*}{ Second order } & 16 & $7.51 \mathrm{E}-3$ & - & $8.01 \mathrm{E}-2$ & - & $1.33 \mathrm{E}-3$ & - & $7.99 \mathrm{E}-5$ & - \\
& 32 & $1.89 \mathrm{E}-3$ & 2.00 & $2.01 \mathrm{E}-2$ & 1.99 & $2.27 \mathrm{E}-4$ & 2.55 & $1.97 \mathrm{E}-5$ & 2.01 \\
& 64 & $4.73 \mathrm{E}-4$ & 2.00 & $5.04 \mathrm{E}-3$ & 2.00 & $4.97 \mathrm{E}-5$ & 2.19 & $4.91 \mathrm{E}-6$ & 2.00 \\
& 128 & $1.18 \mathrm{E}-4$ & 2.00 & $1.26 \mathrm{E}-3$ & 2.00 & $1.20 \mathrm{E}-5$ & 2.05 & $1.20 \mathrm{E}-6$ & 2.00 \\
\hline
\end{tabular}

\subsection{Accuracy test}

Example 6.1. We solve (2.1)-(2.4) and the parameters are taken as

$$
\begin{aligned}
d_{m} & =10^{-2}, \alpha_{l}=0, \alpha_{t}=0, K_{0}=1, T=0.2, \\
\alpha & =k_{c}=k_{s}=\mu=1, f_{p}=f_{I}=0, \rho=1, \\
a_{0} & =0.5, \rho_{s}=10, \gamma=1 .
\end{aligned}
$$

The exact smooth solutions are given as

$$
\begin{aligned}
& p(\mathbf{x}, t)=e^{-t} \cos (2 \pi x) \cos (2 \pi y), \\
& \phi(\mathbf{x}, t)=0.6+t^{2} \sin (2 \pi x) \cos (2 \pi y), \quad c_{f}(\mathbf{x}, t)=0.5+0.1 e^{-t} \sin (2 \pi x) \sin (2 \pi y) .
\end{aligned}
$$

We can calculate the initial conditions and the right hand sides accordingly. Piecewise linear tensor product polynomials are employed in the LDG scheme. We perform accuracy verifications on uniform meshes with $N \times N$ elements over the computational domain $\Omega=[0,1] \times[0,1]$, and compute the numerical approximations until $T=0.2$. Periodic boundary condition is used in this numerical example. The numerical results for the error in $L^{2}$ norm and corresponding order of accuracy are presented in Table 1. From the table, we can observe optimal convergence rates, which verifies the analysis.

The next example is the simulation of real wormhole propagation scenario in petroleum engineering.

\subsection{Wormhole propagation problem}

The computational domain is $\Omega=[0,0.2 \mathrm{~m}] \times[0,0.2 \mathrm{~m}]$. Initial concentration of acid and initial porosity of rock in this domain are set to be $c_{0}=0$ and $\phi_{0}=0.2$, respectively. The acid flow is injected to the porous media from the left boundary with a velocity of $u=0.01 \mathrm{~m} / \mathrm{s}$ and drained out of it from the right boundary with the same velocity. Top and bottom boundary conditions are set to be periodic. The concentration of influx acid is $10 \mathrm{~mol} / \mathrm{m}^{2}$. To observe the wormhole propagation, we set two singular areas with high porosity and permeability on the left boundary with size to be $0.01 \mathrm{~m} \times 0.01 \mathrm{~m}$ : one is $0.05 \mathrm{~m}$ above the bottom with the porosity of 0.4 , and the other $0.1 \mathrm{~m}$ above the bottom with the porosity of 0.6 . The permeability of the two entries is determined by (2.7) which is about $10^{-10} \mathrm{~m}^{2}$ and $10^{-11} \mathrm{~m}^{2}$, respectively. 


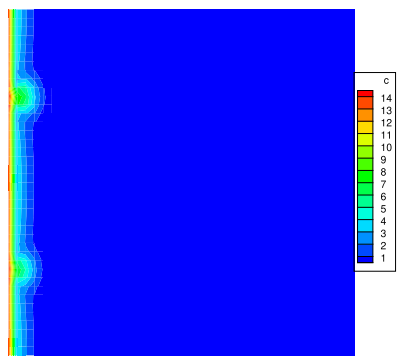

(a)

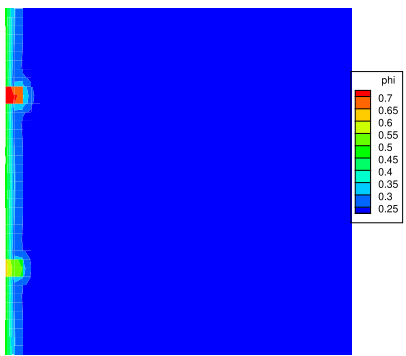

(d)

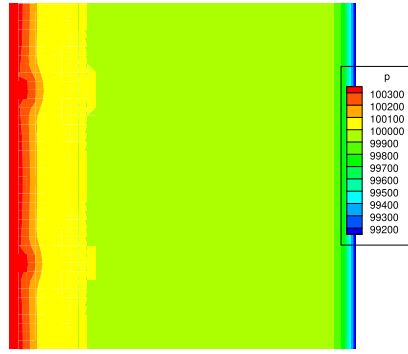

(g)

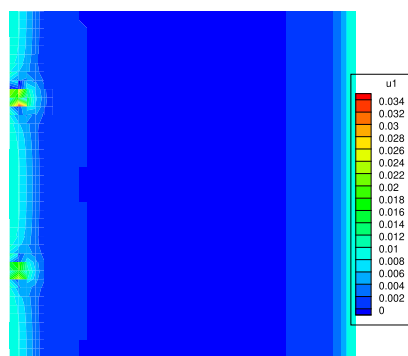

(j)

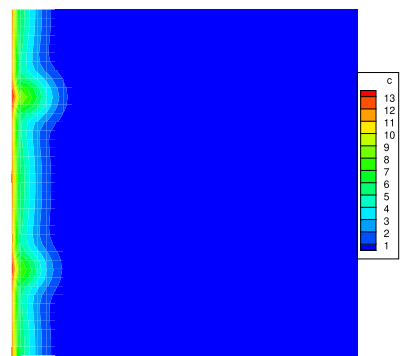

(b)

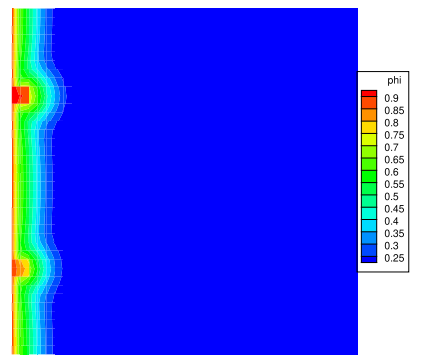

(e)

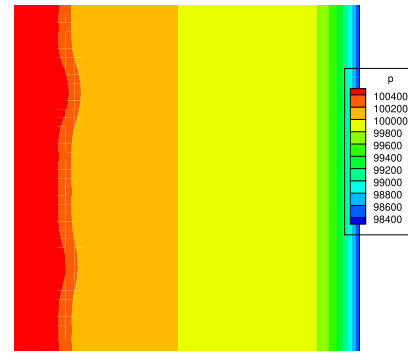

(h)

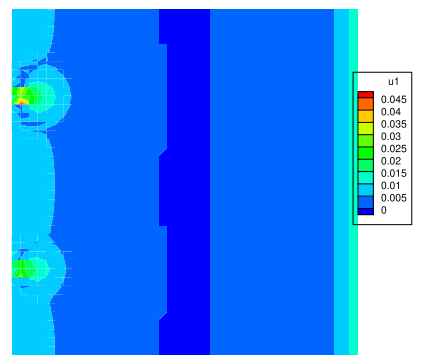

(k)

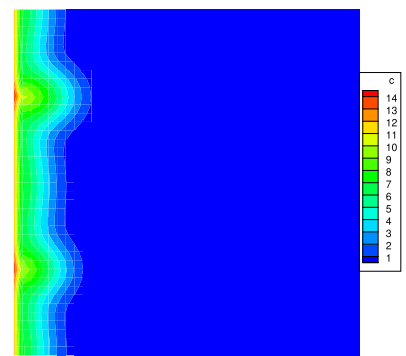

(c)

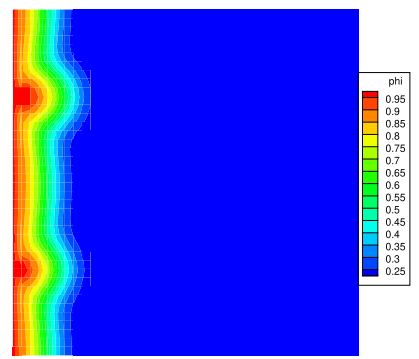

(f)

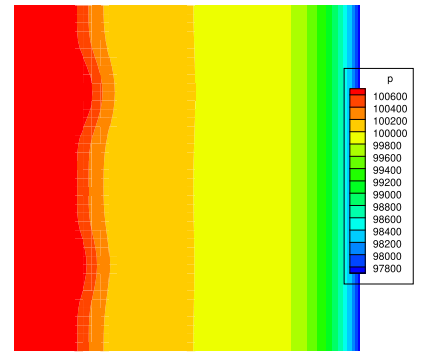

(i)

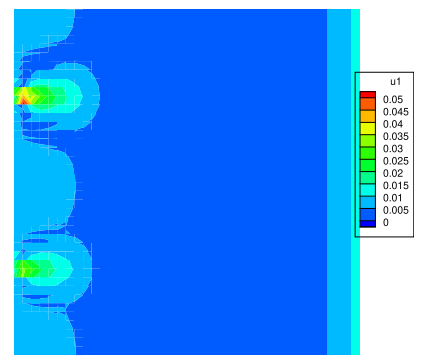

(l)

Figure 1. Example 6.2: $\mathbf{D}(\mathbf{u})=d_{m} \mathbf{I}+|\mathbf{u}|\left\{\alpha_{l} \mathbf{E}(\mathbf{u})+\alpha_{t}(\mathbf{I}-\mathbf{E}(\mathbf{u}))\right\}$, with $d_{m}=10^{-4}, \alpha_{l}=$ $10^{-3}, \alpha_{t}=10^{-4}$. Numerical solutions for concentration, porosity, pressure and velocity along $x$ direction with time evolution are displayed. The computational mesh is composed by $40 \times 40$ elements. The second fully discrete time scheme (4.20)-(4.25) are employed with the time step $\tau=0.1 h$. (a) $c_{f_{h}}$ at $T=5$. (b) $c_{f_{h}}$ at $T=20$. (c) $c_{f_{h}}$ at $T=40$. (d) $\phi_{h}$ at $T=5$. (e) $\phi_{h}$ at $T=20$. (f) $\phi_{h}$ at $T=40$. (g) $p_{h}$ at $T=5$. (h) $p_{h}$ at $T=20$. (i) $p_{h}$ at $T=40$. (j) $u_{h}$ at $T=5$. (k) $u_{h}$ at $T=20$. (l) $u_{h}$ at $T=40$. 
Example 6.2. A real wormhole propagation scenario in petroleum engineering is studied in this example. The parameters are taken as

$$
\begin{aligned}
d_{m} & =10^{-4}, \quad \alpha_{l}=10^{-3}, \quad \alpha_{t}=10^{-4}, \quad \alpha_{l}=0, \quad \alpha_{t}=0, \quad K_{0}=10^{-9} \mathrm{~m}^{2}, \quad T=40 \mathrm{~s}, \\
\alpha & =10 \mathrm{~kg} / \mathrm{mol}, \quad k_{c}=1 \mathrm{~m} / \mathrm{s}, \quad k_{s}=10 \mathrm{~m} / \mathrm{s}, \\
\mu & =10^{-2} \mathrm{~Pa} . \mathrm{s}, \quad f_{I}=f_{p}=0, \quad \rho=1000, \\
a_{0} & =2 \mathrm{~m}^{-1}, \quad \rho_{s}=2500 \mathrm{~kg} / \mathrm{m}^{2}, \quad \gamma=0.01 .
\end{aligned}
$$

In the real wormhole propagation example, the second fully-discrete time scheme (4.20)-(4.25) is employed, and the time step is chosen as $\tau=0.1 h$ with uniform mesh size $h$. The LDG discretization with linear polynomial is used in this example. The contour plots of concentration of acid, porosity of rock and pressure on a uniform mesh with $40 \times 40$ elements with time evolution are shown in Figure 1 . We can clearly see the wormhole generate and grow with time.

\section{Concluding Remarks}

In this paper, we applied the LDG spatial discretization coupled with two time integrations to wormhole propagations. We defined a new auxiliary variable including both the convection and diffusion terms. Moreover, we applied a special time integration of the porosity, leading to physically relevant numerical approximations and controllable growth rate of the porosity. Thanks to the above techniques, we obtained the stability of the first order time integration. We also proved the optimal error estimates for the pressure, velocity, porosity and concentration under different norms up to second order time integration under weak temporal-spatial conditions. Numerical experiments were also given to verify the theoretical results. In the future work, we will design more efficient and higher order methods for this problem.

Acknowledgements. The first author and the third author were supported by the Fundamental Research Funds for the Central Universities 20CX05011A and the Major Scientific and Technological Projects of CNPC under Grant ZD2019183-008. The last author was supported by NSF grant DMS-1818467. The third author was supported by NSFC grant 11801569, Natural Science Foundation of Shandong Province (CN) grant ZR2018BA011.

\section{REFERENCES}

[1] O. Akanni, H. Nasr-El-Din and D. Gusain, A computational Navier-Stokes fluid-dynamics-simulation study of wormhole propagation in carbonate-matrix acidizing and analysis of factors influencing the dissolution process. SPE J. 22 (2017) 187962.

[2] F. Bassi and S. Rebay, A high-order accurate discontinuous finite element method for the numerical solution of the compressible Navier-Stokes equations. J. Comput. Phys. 131 (1997) 267-279.

[3] P. Castillo, B. Cockburn, I. Perugia and D. Schötzau, Superconvergence of the local discontinuous Galerkin method for elliptic problems on cartesian grids. SIAM J. Numer. Anal. 39 (2001) 264-285.

[4] P. Ciarlet, The Finite Element Method for Elliptic Problem. North Holland (1975).

[5] B. Cockburn and C.-W. Shu, The local discontinuous Galerkin method for time-dependent convection-diffusion systems. SIAM J. Numer. Anal. 35 (1998) 2440-2463.

[6] C. Fredd and H. Fogler, Influence of transport and reaction on wormhole formation in porous media. Fluid Mech. Transp. Phenom. 44 (1998) 1933-1949.

[7] F. Golfier, C. Zarcone, B. Bazin, R. Lenormand, D. Lasseux and M. Quintard, On the ability of a Darcy-scale model to capture wormhole formation during the dissolution of a porous medium. J. Fluid Mech. 457 (2002) 213-254.

[8] T.H. Gronwall, Note on the derivative with respect to a parameter of the solutions of a system of differential equations. Ann. Math. 20 (1919) 292-296.

[9] H. Guo, F. Yu and Y. Yang, Local discontinuous Galerkin method for incompressible miscible displacement problem in porous media. J. Sci. Comput. 71 (2017) 615-633.

[10] H. Guo, L. Tian, Z. Xu, Y. Yang and N. Qi, High-order local discontinuous Galerkin method for simulating wormhole propagation. J. Comput. Appl. Math. 350 (2019) 247-261.

[11] J. Kou, S. Sun and Y. Wu, Mixed finite element-based fully conservative methods for simulating wormhole propagation. Comput. Methods Appl. Mech. Eng. 298 (2016) 279-302. 
[12] X. Li and H. Rui, Characteristic block-centered finite difference method for simulating incompressible wormhole propagation. Comput. Math. App. 73 (2017) 2171-2190.

[13] X. Li and H. Rui, Block-centered finite difference method for simulating compressible wormhole propagation. J. Sci. Comput. 74 (2017) 1115-1145.

[14] X. Li and H. Rui, A fully conservative finite difference method for simulating Darcy-Forchheimer compressible wormhole propagation. Numer. Algorithms (2018).

[15] X. Li, C.-W. Shu and Y. Yang, Local discontinuous Galerkin method for the Keller-Segel chemotaxis model. J. Sci. Comput. 73 (2017) 943-967.

[16] M. Liu, S. Zhang, J. Mou and F. Zhou, Wormhole propagation behavior under reservoir condition in carbonate acidizing. Transp. Porous Media 96 (2013) 203-220.

[17] P. Maheshwari and V. Balakotaiah, 3D Simulation of Carbonate Acidization with HCl: Comparison with Experiments. Society of Petroleum Engineers (2013).

[18] S. Mauran, L. Rigaud and O. Coudevylle, Application of the carman-kozeny correlation to a highporosity and anisotropic consolidated medium: The compressed expanded natural graphite Transp. Porous Media 43 (2001) 355-376.

[19] M. Panga and M. Ziauddin, Two-scale continuum model for simulation of wormholes in carbonate acidization. AIChE J. 51 (2005) 3231-3248

[20] A. Smirnov, K. Fedorov and A. Shevelev, Modeling the acidizing of a carbonate formation. Fluid Dyn. 45 (2010) $779-786$.

[21] P. Szymczak and A. Ladd, Wormhole formation in dissolving fractures. J. Gophys. Res. 114 (2009) B06203.

[22] L. Tian, H. Guo, R. Jia and Y. Yang, An $h$-adaptive local discontinuous galerkin method for simulating wormhole propagation with Darcy-Forcheiner model. J. Sci. Comput. 82 (2020) 43.

[23] H. Wang, C.-W. Shu and Q. Zhang, Stability and error estimates of local discontinuous Galerkin methods with implicit-explicit time-marching for advection-diffusion problems. SIAM J. Numer. Anal. 53 (2015) 206-227.

[24] H. Wang, C.-W. Shu and Q. Zhang, Stability analysis and error estimates of local discontinuous Galerkin methods with implicit-explicit time-marching for nonlinear convection-diffusion problems. Appl. Math. Comput. 272 (2016) $237-258$.

[25] H. Wang, S. Wang, Q. Zhang and C.-W. Shu, Local discontinuous Galerkin methods with implicit-explicit time marching for multi-dimensional convectiondiffusion problems. ESAIM: M2AN 50 (2016) 1083-1105.

[26] H. Wang, J. Zheng, F. Yu, H. Guo and Q. Zhang, Local Discontinuous Galerkin method with implicit-explicit time marching for incompressible miscible displacement problem in porous media. J. Sci. Comput. 78 (2018) 1-28.

[27] H. Wang, Y. Liu, Q. Zhang and C.-W. Shu, Local discontinuous Galerkin methods with implicit-explicit time-marching for time-dependent incompressible fluid flow. Math. Comput. 88 (2019) 91-121.

[28] H. Wang, Q. Zhang, S. Wang and C.W. Shu, Local discontinuous Galerkin methods with explicit-implicit-null time discretizations for solving nonlinear diffusion problems. Sci. China (Math.) 063 (2020) 183-204.

[29] W. Wei, A. Varavei and K. Sepehrnoori, Modeling and analysis on the effect of two-phase flow on wormhole propagation in carbonate acidizing. SPE J. 22 (2017).

[30] Y. Wu, A. Salama and S. Sun, Parallel simulation of wormhole propagation with the Darcy-Brinkman-Forchheimer framework. Comput. Geotech. 69 (2015) 564-577.

[31] Y. Xu and C.-W. Shu, Local discontinuous Galerkin methods for nonlinear Schr odinger equations. J. Comput. Phys. 205 (2005) 72-97.

[32] Y. Xu and C.-W. Shu, Error estimates of the semi-discrete local discontinuous Galerkin method for nonlinear convectiondiffusion and KdV equations. Comput. Methods Appl. Mech. Eng. 196 (2007) 3805-3822.

[33] Z. Xu, Y. Yang and H. Guo, High-Order bound-preserving discontinuous Galerkin methods for wormhole propagation on triangular meshes. J. Comput. Phys. 390 (2019) 323-341.

[34] J. Yan and C.-W. Shu, Local discontinuous Galerkin methods for partial differential equations with higher order derivatives. J. Sci. Comput. 17 (2002) 27-47.

[35] J. Yan and C.-W. Shu, A local discontinuous Galerkin method for KdV type equations. SIAM J. Numer. Anal. 40 (2002) 769-791.

[36] F. Yu, H. Guo, N. Chuenjarern and Y. Yang, Conservative local discontinuous Galerkin method for compressible miscible displacements in porous media. J. Sci. Comput. 73 (2017) 1249-1275.

[37] J. Zhang, X. Shen, H. Guo, H. Fu and H. Han, Characteristic splitting mixed finite element analysis of compressible wormhole propagation. Appl. Numer. Math. 147 (2020) 66-87.

[38] C. Zhao, B.E. Hobbs, P. Hornb, A. Ord, S. Peng and L. Liu, Theoretical and numerical analyses of chemical-dissolution front instability in fluid-saturated porous rocks. Int. J. Numer. Anal. Methods Geomech. 32 (2008) 1107-1130. 